\title{
Nucleação na formação de estruvita: estado da arte
}

\author{
Nucleation in the formation of struvite: state of art \\ Dione Mari Morita** ${ }^{10}$, Renan de Luca Avila' ${ }^{\circledR}$, Fernando Ngan Aidar ${ }^{1}(\mathbb{C}$
}

口-

\begin{abstract}
RESUMO
Estimativas recentes indicam que a duração de toda a reserva de rochas fosfatadas do planeta não ultrapassa 400 anos e que a produção de fertilizantes nitrogenados é insustentável energeticamente. Atualmente, o tratamento de esgoto está sendo visto como uma unidade de recuperação de recursos (água, energia e nutrientes) em vez de uma de final de tubo. Portanto, as tecnologias de recuperação devem ser priorizadas em relação às de remoção. Estruvita (fosfato deamônio e magnésio) é um cristal que pode ser produzido a partir do esgoto doméstico e tem maior eficiência agrícola do que os fertilizantes convencionais. Apesar de a cristalização ser um tema bastante estudado em todo o mundo e extremamente desenvolvido tecnologicamente, sua utilização na engenharia sanitária ainda não é comum, especialmente no Brasil. Um dos assuntos menos pesquisados na formação da estruvita é a nucleação. Portanto, o presente artigo traz uma revisão bibliográfica crítica e atual e mostra as lacunas no conhecimento sobre esse assunto.
\end{abstract}

Palavras-chave: esgoto; recuperação de nutrientes; estruvita; termodinâmica; nucleação.

$\square$

\section{INTRODUÇÃO}

A população mundial cresce continuamente e, portanto, garantir a oferta adequada de alimentos é uma prioridade (ITPS, 2015). De acordo com Erisman et al. (2008) e Alexandratos e Bruinsma (2012), o aumento no consumo de alimento per capita e as alterações de dieta nos países subdesenvolvidos e em desenvolvimento, as quais tendem a um maior consumo de carne, serão os principais fatores que impactarão a demanda mundial de alimentos no futuro. Como consequência, prevê-se que ocorrerá aumento no uso de fertilizantes.

Segundo Cordell e White (2014) e Heckenmüller, Narita e Klepper (2014), embora a estimativa sobre a exaustão das reservas de fósforo no mundo varie muito, ela não ultrapassará os 400 anos (SMIT ET AL., 2009; VAN KAUWENBERGH, 2010; CALVO; 55 VALERO; VALERO, 2017).

Quanto ao nitrogênio, apesar de a atmosfera ser uma fonte ilimitada, a eficiência de transformação do gás nitrogênio em fertilizantes é muito baixa e os requisitos energéticos e os impactos ambientais são altos, mesmo com os avanços ocorridos nas últimas décadas no processo Haber-Bosch (GALLOWAY et al., 2003; CHERKASOV; IBHADON; FITZPATRICK, 2015).

\begin{abstract}
Recent estimation for the duration of global phosphate reserves does not exceed 400 years and the production of nitrogen fertilizers is energetically unsustainable. Nowadays, wastewater treatment plants have been seen as resource recovery facilities (water, energy and nutrients) instead of "end-ofpipe" utilities. Thus, nutrient-recovery technologies should be prioritized over the removal ones. Struvite (phosphate of ammonium and magnesium) is a crystal, produced from sewage, with a higher agricultural efficiency when compared to conventional fertilizers. Despite the crystallization is a topic extensively studied worldwide and highly technologically developed, its use in sanitary engineering is still not common, especially in Brazil. As the aspects related to struvite nucleation have been insufficiently studied, this paper presents a critical and updated literature review, and shows the gaps in the current knowledge about this topic.
\end{abstract}

Keywords: sewage; nutrients recovery; struvite; thermodynamics; nucleation . 
(THISTLETON et al., 2002), entretanto as dosagens de sais de ferro e de alumínio são altas; a produção de lodo aumenta em até 95\% (YEOMAN et al., 1988; SHU et al., 2006); a geração de metano reduz em até 5,5\% (SMITH; CARLIELL-MARQUET, 2008); e o lodo (fosfatos de ferro ou de alumínio) apresenta dificuldade de desaguamento e de liberação de fósforo no solo (MELIA et al., 2017; DESMIDT et al., 2015).

Outra forma de recuperar o fósforo presente no esgoto doméstico para a aplicação na agricultura é a precipitação como estruvita $\left(\mathrm{MgNH}_{4} \mathrm{PO}_{4} \cdot 6 \mathrm{H}_{2} \mathrm{O}\right)$. Segundo Adnan, Mavinic e Koch (2003), Britton et al. (2005) e Cornel e Schaum (2009), concentrações de fósforo menores do que 40 a $60 \mathrm{mgP}-\mathrm{PO}_{4} \cdot \mathrm{L}^{-1}$ são bastante desfavoráveis para a precipitação química da estruvita. Por essa razão, ela é produzida por meio da urina pura, das correntes líquidas provenientes do processo de digestão anaeróbia e de desaguamento do lodo de sistemas EBPR ou das cinzas do lodo incinerado.

A estruvita apresenta as seguintes vantagens sobre os demais fertilizantes encontrados no mercado (BRIDGER; SALUTSKY; STAROSTKA, 1962; WANG et al., 2005; BATTISTONI et al., 2005; MÜNCH; BARR, 2001; UENO; FUJII, 2001; UYSAL; YILMAZEL; DEMIRER, 2010):

- sua dissolução é lenta e, por isso, as aplicações são menos frequentes - as plantas conseguem absorver os nutrientes antes que estes sejam lavados pelo escoamento superficial;

- as plantas não sofrem problemas relacionados à hipersaturação de nutrientes;

- os fertilizantes de origem mineral costumam possuir teores de metais pesados maiores que os encontrados na estruvita;

- os macronutrientes essenciais N, P e Mg são introduzidos simultaneamente no solo;

- pelo fato de ser um fertilizante de liberação lenta, reduz-se o problema mundial de eutrofização dos corpos d'água superficiais.

Embora a estruvita apresente tais vantagens, ela ainda não é produzida em larga escala pelas seguintes razões:

- grandes quantidades de magnésio e de álcali são necessárias, o que aumenta os custos operacionais;

- é difícil obter estruvita com alta pureza do esgoto sanitário ou de águas residuárias industriais;

- o mercado para a estruvita não está totalmente estabelecido;

- faltam incentivos legais e econômicos para a reciclagem do fósforo visando a segurança alimentar no futuro (HAO et al., 2013; DESMIDT et al., 2015; HUANG et al., 2015; KATAKI et al., 2016a; 2016b).

\section{FORMAÇÃO DA ESTRUVITA}

A estruvita é produzida em duas etapas: nucleação e crescimento cristalino. Por conta da complexidade do assunto, neste artigo, só será discutida a primeira etapa.
A nucleação corresponde à aparição do primeiro núcleo de estruvita, que se forma em soluções supersaturadas conforme a Reação 1,

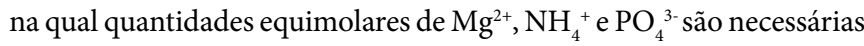
para produzir $\mathrm{MgNH}_{4} \mathrm{PO}_{4} \cdot 6 \mathrm{H}_{2} \mathrm{O}$ (HANHOUN et al., 2011; OHLINGER; YOUNG; SCHROEDER, 1998; RAHAMAN; ELLIS; MAVINIC, 2008; OUCHAH et al., 2014; SONG et al., 2007).

$\mathrm{Mg}^{2+}+\mathrm{NH}_{4}^{+}+\mathrm{PO}_{4}^{3-}+6 \mathrm{H}_{2} \mathrm{O} € \mathrm{MgNH}_{4} \mathrm{PO}_{4} \cdot 6 \mathrm{H}_{2} \mathrm{O}(\mathrm{s})$

Entretanto, na literatura, não há consenso sobre os mecanismos envolvidos nessa formação. Segundo Abbona e Boistelle (1979), inicialmente, parte do magnésio é hidratado $-\mathrm{Mg}\left(\mathrm{H}_{2} \mathrm{O}\right)_{6}^{2+}$ - e se liga ao tetraedro $\mathrm{PO}_{4}^{3-}$ por atração eletrostática. $\mathrm{O} \mathrm{Mg}^{2+}$ não hidratado reage com o $\mathrm{HPO}_{4}^{2-}$, produzindo $\mathrm{MgHPO}_{4}$, que, por sua vez, é desprotonado e transformado em $\mathrm{MgPO}_{4}^{-}$. Formam-se pontes de hidrogênio entre os íons $\mathrm{PO}_{4}^{3-}, \mathrm{Mg}\left(\mathrm{H}_{2} \mathrm{O}\right)_{6}^{2+}, \mathrm{MgPO}_{4}^{-}$e $\mathrm{NH}_{4}^{+}$, mantendo-os juntos e produzindo a estrutura cristalina da estruvita. Outros pesquisadores afirmam que ocorre a Reação 2, em razão da constatação de que há um decréscimo de pH após a formação da estruvita (ALI, 2007; HAO et al., 2008; LE CORRE et al., 2009; MPOUNTAS; PAPADAKIS; KOUTSOUKOS, 2017).

$\mathrm{Mg}^{2+}+\mathrm{NH}_{4}^{+}+\mathrm{H}_{n} \mathrm{PO}_{4}^{n-3}+6 \mathrm{H}_{2} \mathrm{O} \otimes \mathrm{MgNH}_{4} \mathrm{PO}_{4} \cdot 6 \mathrm{H}_{2} \mathrm{O}(\mathrm{s})+n \mathrm{H}^{+}$

com $\mathrm{n}=0,1$ ou 2 de acordo com o $\mathrm{pH}$

Já Tansel, Lunn e Monje (2018) acreditam que, como o $\mathrm{Mg}^{2+}$ tem maior afinidade à água do que as espécies de nitrogênio e fósforo, ele é hidratado rapidamente, produzindo o octaedro $\mathrm{Mg}\left(\mathrm{H}_{2} \mathrm{O}\right)_{6}^{2+}$. A forte interação entre o $\mathrm{Mg}^{2+}$ e o $\mathrm{HPO}_{4}^{2-}$ ou o $\mathrm{PO}_{4}^{3-}$ pode criar condições seletivas para a entrada de $\mathrm{NH}_{3_{(a q)}}$ na estrutura cristalina, por este ser menor e ter maior mobilidade do que o íon $\mathrm{NH}_{4}^{+}$. O $\mathrm{NH}_{3_{(a q)}}$, por sua vez, reage com o $\mathrm{HPO}_{4}^{2-}$, modificando a ligação $\mathrm{H}-\mathrm{PO}_{4}, \mathrm{o}$ que resulta na formação de pontes de hidrogênio do octaedro com os íons $\mathrm{NH}_{4}^{+}$e $\mathrm{PO}_{4}^{3-}$. Essas pontes e o aumento do tamanho do $\mathrm{NH}_{3_{(a q)}}$, pois se transformou em $\mathrm{NH}_{4}^{+}$, estabilizam o cristal. Esse mecanismo explicaria a produção de estruvita em valores de $\mathrm{pH}$ mais altos, situação na qual a concentração de $\mathrm{PO}_{4}^{3-}$ é elevada e a de $\mathrm{NH}_{4}^{+}$, baixa. Conforme esses pesquisadores, a Reação 2 ocorre apenas em locais que sofrem influência da pressão, tais como em tubulações de digestores anaeróbios, ou quando as concentrações dos íons são altas, como, por exemplo, nos rins.

Lin, Bassin e Van Loosdrecht (2012) descobriram que os polímeros extracelulares insolúveis (EPS) induzem a formação de estruvita em sistemas de lodo ativado granular. Os fosfatos das bactérias acumuladoras de fósforo (PAO) são liberados na fase anaeróbia e utilizados pelas PAO no estágio aeróbio. Os grânulos aeróbios têm polímeros extracelulares insolúveis parecidos com o 
alginato (EPS) que possuem cargas superficiais negativas, atraindo cátions do meio, tais como o $\mathrm{Ca}^{2+}, \mathrm{o} \mathrm{K}^{+}, \mathrm{o} \mathrm{Mg}^{2+}$. $\mathrm{O}$ cálcio tem maior afinidade com o EPS e participa na formação da estrutura do gel. O potássio tem interação mais fraca e, portanto, pode trocar com o $\mathrm{NH}_{4}^{+}$. Propicia-se, assim, a nucleação de K-estruvita e/ou estruvita na superfície do grânulo aeróbio, a depender das concentrações disponíveis de $\mathrm{K}^{+}$e $\mathrm{NH}_{4}^{+}$no meio aquoso. A partir dessa descoberta, pesquisadores mostraram que era possível gerar estruvita biologicamente (SOARES et al., 2014; RIVADENEYRA et al., 2014; SIMÕES et al., 2018). Essa nova rota apresenta as seguintes vantagens: não é necessária uma concentração de fósforo acima de 40 a $60 \mathrm{mgP}-\mathrm{PO}_{4} \cdot \mathrm{L}^{-1}$; os cristais produzidos são grandes; não se usa produto químico; consegue-se concentração abaixo de $2 \mathrm{mgP}$ $-\mathrm{PO}_{4} \cdot \mathrm{L}^{-1}$ no efluente tratado para concentrações de 5 a $30 \mathrm{mgP}$ $-\mathrm{PO}_{4} \cdot \mathrm{L}^{-1}$ na água residuária bruta. $\mathrm{O}$ alto tempo de detenção (de 3 a 10 dias) é a principal desvantagem. Recentemente, Simões et al. (2018) mostraram que era possível produzir maior quantidade de estruvita biologicamente do que quimicamente no intervalo de $\mathrm{pH}$ entre 7,3 e 7,8; acima de 8,3, predominava o mecanismo químico.

\section{Especiação do fósforo, do nitrogênio e do magnésio}

Conforme visto, nem todas as espécies de magnésio, de fósforo e de nitrogênio são usadas na formação da estruvita. Valores mais baixos de $\mathrm{pH}$ favorecem a formação do ácido fosfórico na solução, $\mathrm{H}_{3} \mathrm{PO}_{4}, \mathrm{o}$ qual se transforma gradualmente nos íons $\mathrm{H}_{2} \mathrm{PO}_{4}^{-}, \mathrm{HPO}_{4}{ }^{2-}$ e $\mathrm{PO}_{4}^{3-}$ conforme se eleva o $\mathrm{pH}$. Quanto ao nitrogênio, em valores de $\mathrm{pH}$ menores que 9,50, há a predominância do íon amônio $\left(\mathrm{NH}_{4}^{+}\right)$, enquanto em $\mathrm{pH}$ mais elevado, há a formação da amônia $\left(\mathrm{NH}_{3}\right)$. Da mesma maneira, o íon $\mathrm{Mg}^{2+}$ se transforma no complexo $\mathrm{MgOH}^{+}$conforme se eleva o $\mathrm{pH}$. Não é só o pH que determina as concentrações das espécies; a temperatura, a intensidade iônica e as concentrações dos reagentes também têm influência significativa. A título de ilustração, a Figura 1 mostra a concentração de cada uma das espécies de fósforo em função do $\mathrm{pH}$ para diferentes temperaturas, intensidades iônicas e concentrações totais desses elementos.

Além das variáveis discutidas anteriormente, as impurezas presentes nas águas residuárias têm significativa influência na formação da estruvita, que está associada ao aumento ou à diminuição da solubilidade do mineral; à alteração no tempo de indução; à mudança na morfologia e no tamanho do cristal; ao efeito do íon comum; à competição pelos íons que formam a estruvita e/ou à substituição dos íons na malha cristalina, que reduz a sua qualidade (ANDRADE; SCHUILING, 2001). Pesquisadores têm estudado a interferência do sódio, sulfatos e carbonatos (KABDASZLI; PARSONS; TÜNAY, 2006); do ácido lático (KOZIK et al., 2011); do cobre (HUTNIK et al., 2013); do zinco (HUTNIK et al., 2016); do alumínio, do cobre, do ferro, do potássio e do zinco (KOZIK et al., 2013) presentes em águas residuárias industriais e lixiviado de aterro sanitário, mas, para o caso do esgoto, os artigos se concentram no cálcio. A Tabela 1 mostra os resultados de estudos que foram realizados para avaliar tal interferência. Nota-se que as principais variáveis são a relação $\mathrm{Ca}^{2+}: \mathrm{Mg}^{2+}$ e o pH. Como o cálcio compete com o magnésio, quanto maior a relação $\mathrm{Ca}^{2+}: \mathrm{Mg}^{2+} \mathrm{e}$ maior o pH, situação na qual ocorre a maior volatilização da amônia, maior a inibição à formação da estruvita. Outro forte inibidor, que tem sido estudado nos últimos anos, é o ferro (YAN; SHIH, 2016; FLORES-ALSINA et al., 2016).

Para o cálculo das concentrações das espécies, é necessário conhecer as reações que ocorrem no meio, levando em consideração os interferentes. Foi realizado um levantamento das reações consideradas pelos pesquisadores que estudaram a formação e a dissolução da estruvita. Os resultados são mostrados na Tabela 2. Verifica-se que cada pesquisador considerou uma série diferente de reações, o que torna difícil a comparação direta dos resultados obtidos por diferentes autores.

Os valores das constantes de equilíbrio (k) das reações são determinados normalmente em $25^{\circ} \mathrm{C}(298 \mathrm{~K})$. Vários autores utilizam a equação de Van’t Hoff (Equação 1) para corrigi-los para outras temperaturas.

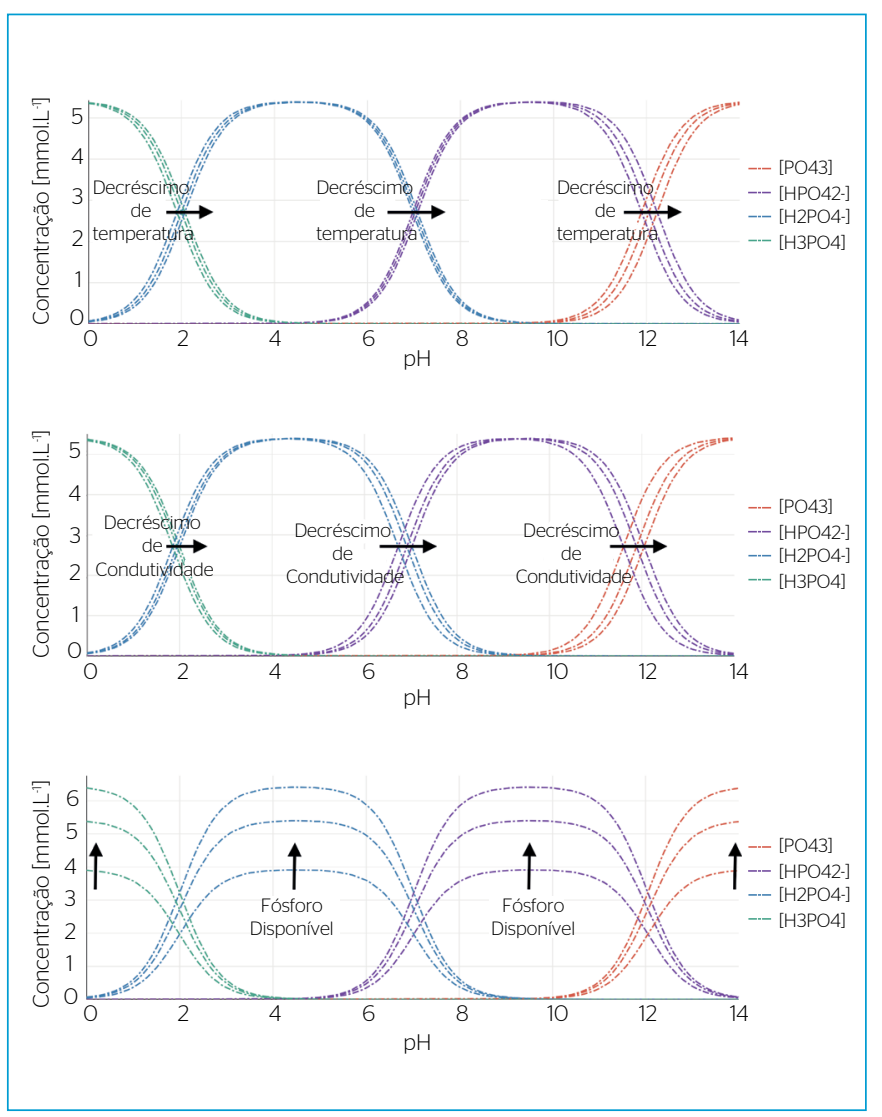

Figura 1 - Variação das concentrações das espécies de fósforo em função do $\mathrm{pH}$ : (A) em diferentes temperaturas; (B) em diferentes intensidades iônicas; (C) em diferentes concentrações totais de fósforo. 


\begin{tabular}{|c|c|c|c|c|c|c|c|c|}
\hline 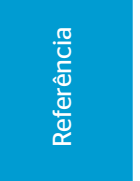 & 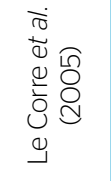 & 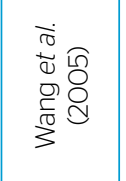 & 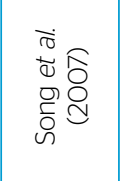 & 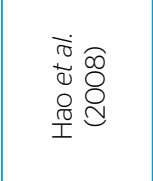 & 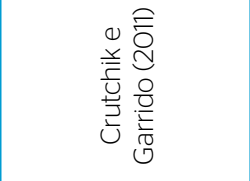 & 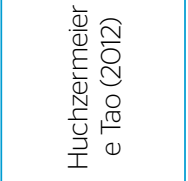 & 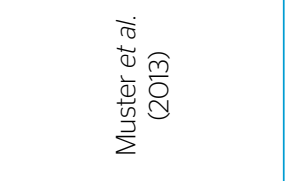 & 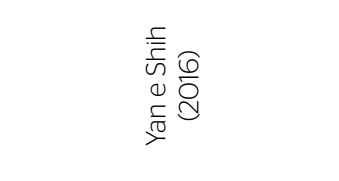 \\
\hline 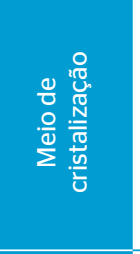 & 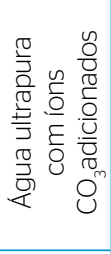 & 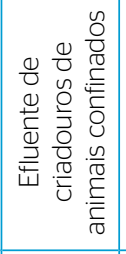 & 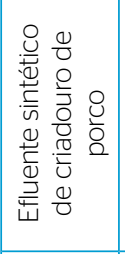 & 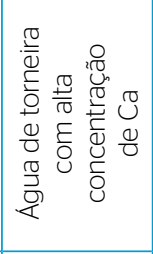 & 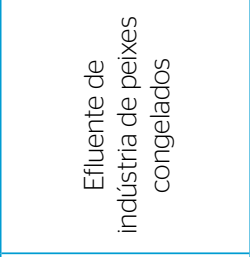 & 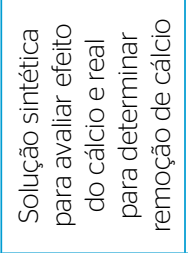 & 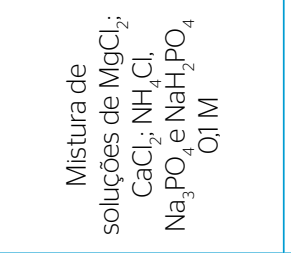 & 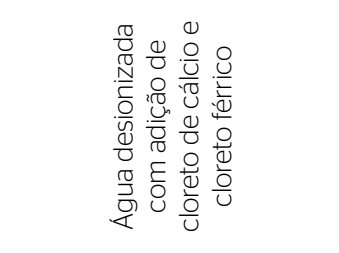 \\
\hline 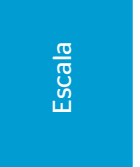 & 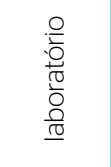 & $\begin{array}{l}.0 \\
\frac{0}{0} \\
\frac{0}{0} \\
\frac{0}{0} \\
\frac{0}{10}\end{array}$ & 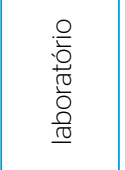 & 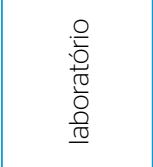 & $\frac{\circ}{\stackrel{0}{\overline{0}}}$ & 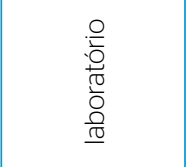 & $\begin{array}{l}\frac{0}{2} \\
\frac{0}{0} \\
\frac{\pi}{0} \\
\frac{0}{0} \\
\frac{0}{10}\end{array}$ & 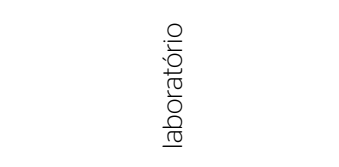 \\
\hline 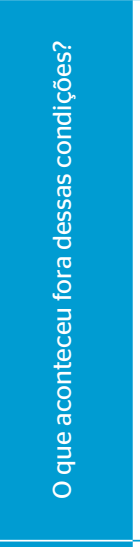 & 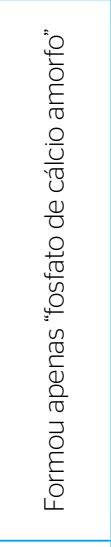 & 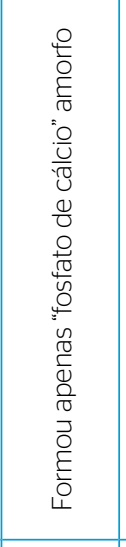 & 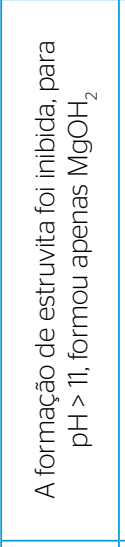 & 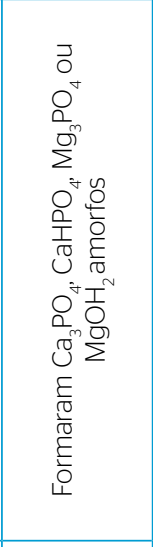 & 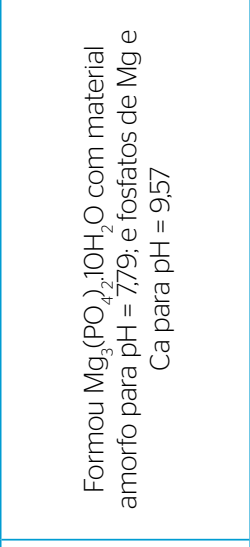 & 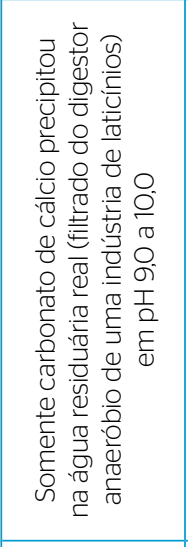 & 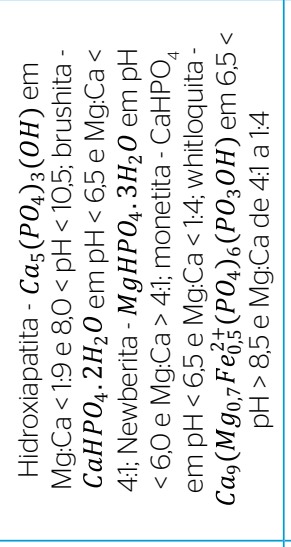 & 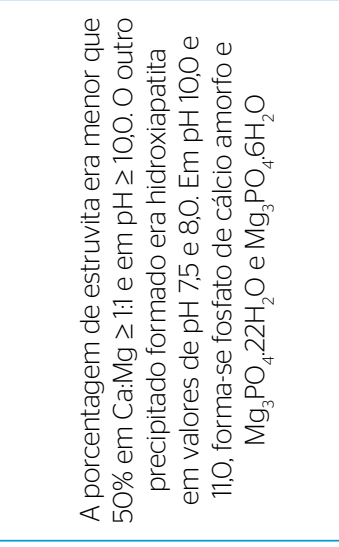 \\
\hline 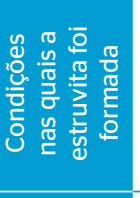 & 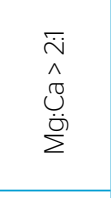 & 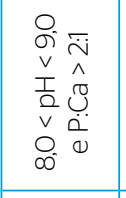 & $\begin{array}{l}\bar{N} \\
\hat{0} \\
u \\
\dot{m}\end{array}$ & 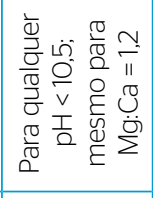 & 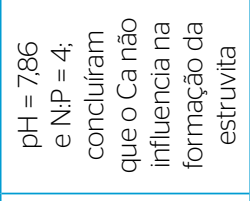 & 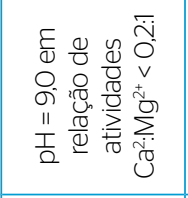 & 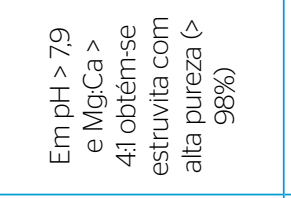 & 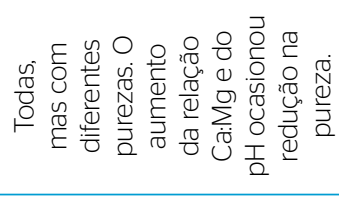 \\
\hline 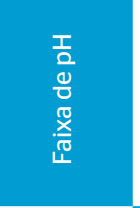 & \&. & 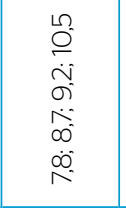 & $\stackrel{\llcorner}{\sigma}$ & 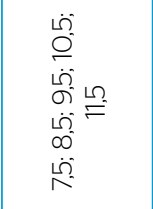 & 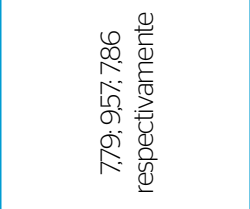 & $\begin{array}{l}\stackrel{0}{0} \\
\frac{0}{0} \\
\stackrel{1}{1}\end{array}$ & 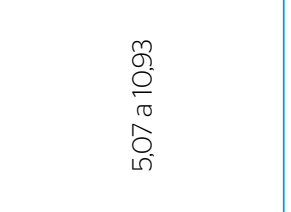 & 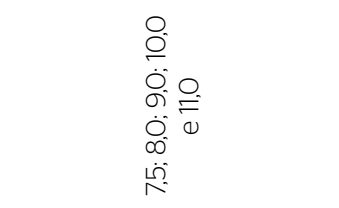 \\
\hline 정 & 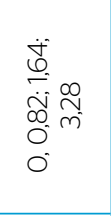 & 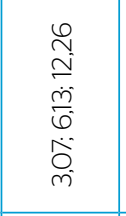 & 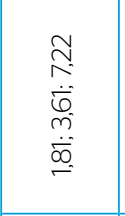 & $\stackrel{\bar{v}}{\bar{v}}$ & 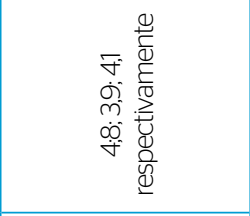 & 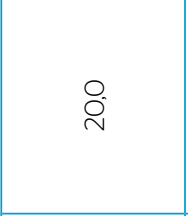 & $\begin{array}{l}0 \\
\varnothing \\
\sigma \\
0\end{array}$ & 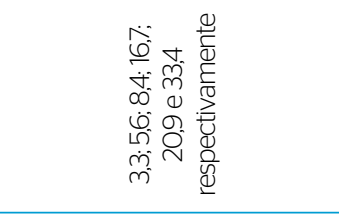 \\
\hline 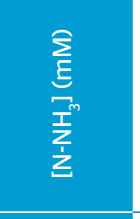 & $\stackrel{\stackrel{\infty}{\sim}}{m}$ & $\begin{array}{l}\stackrel{U}{\mathbb{I}} \\
\text {. }\end{array}$ & 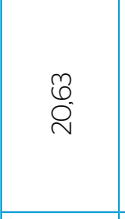 & $\stackrel{N}{\curvearrowright}$ & 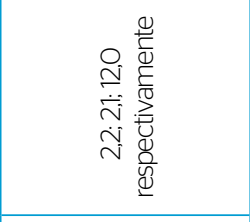 & $\hat{\phi^{\circ}}$ & ㅇ. & $\underline{\underline{\theta}}$ \\
\hline 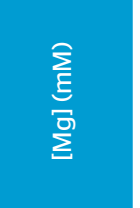 & $\stackrel{\Xi}{\stackrel{\Xi}{\sigma}}$ & 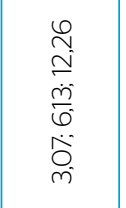 & $\underset{\bar{m}}{\bar{m}}$ & $\stackrel{\llcorner}{\sim}$ & 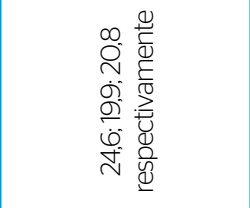 & $\stackrel{\stackrel{L}{\perp}}{=}$ & $\begin{array}{l}\mathscr{\theta} \\
\sigma \\
\sigma \\
0 \\
0\end{array}$ & $\underline{\varphi}$ \\
\hline 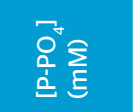 & $\underset{Ð}{\stackrel{\Xi}{C}}$ & $\frac{m}{6}$ & $\stackrel{\infty}{\sim}$ & $\stackrel{\circ}{\text { m. }}$ & 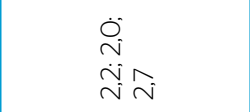 & $\hat{0}$ & $\stackrel{\stackrel{\llcorner}{\llcorner}}{\curvearrowleft}$ & $\underline{\hat{\theta}}$ \\
\hline
\end{tabular}


Tabela 2 - Reações consideradas pelos pesquisadores que estudaram os aspectos termodinâmicos da formação e dissolução da estruvita.

\begin{tabular}{|c|c|c|}
\hline Reação & $\begin{array}{l}\operatorname{logk} \text { ou } \log \mathrm{K}_{\mathrm{pS}} \\
\text { onde especificado }\end{array}$ & Referência \\
\hline $\mathrm{H}_{2} \mathrm{O} \leftrightarrow \mathrm{H}^{+}+\mathrm{OH}^{-}$ & 14,0 & $\begin{array}{l}\text { Bhuyian, Mavinic e Beckie (2007), Babic-Ivancic et al. } \\
\text { (2002), Hanhoun et al. (2011), Rahaman et al. (2014) }\end{array}$ \\
\hline $\mathrm{NH}_{3}+\mathrm{H}^{+} \leftrightarrow \mathrm{NH}_{4}^{+}$ & 9,24 & $\begin{array}{l}\text { Webb e Ho (1991), Babic-Ivancic et al. (2002), Bhuyian, } \\
\text { Mavinic e Beckie (2007), Doyle e Parsons (2002), } \\
\text { Bouropoulos e Koutsoukos (2000), Ronteltap, Maurer e } \\
\text { Gujer (2007), Hanhoun et al. (2011), Rahaman et al. (2014) }\end{array}$ \\
\hline $\mathrm{HCO}_{3}^{-} \leftrightarrow \mathrm{CO}_{3}^{2-}+\mathrm{H}^{+}$ & $-10,33$ & Ronteltap, Maurer e Gujer (2007) \\
\hline $\mathrm{H}^{+}+\mathrm{PO}_{4}^{3-} \leftrightarrow \mathrm{HPO}_{4}^{2-}$ & 12,32 & $\begin{array}{l}\text { Webb e Ho (1991), Bhuyian, Mavinic e Beckie (2007), Babic- } \\
\text { Ivancic et al. (2002), Doyle e Parsons (2002), Ronteltap, Maurer } \\
\text { e Gujer (2007), Hanhoun et al. (2011), Rahaman et al. (2014) }\end{array}$ \\
\hline $\mathrm{H}_{3} \mathrm{PO}_{4} \leftrightarrow \mathrm{H}_{2} \mathrm{PO}_{4}^{-}+\mathrm{H}^{+}$ & $-2,1$ & $\begin{array}{l}\text { Webb e Ho (1991), Bouropoulos e Koutsoukos (2000), Babic- } \\
\text { Ivancic et al. (2002), Hanhoun et al. (2011), Rahaman et al. (2014) }\end{array}$ \\
\hline $\mathrm{HPO}_{4}^{2-}+2 \mathrm{H}^{+} \leftrightarrow \mathrm{H}_{3} \mathrm{PO}_{4}$ & 9,37 & Bhuyian, Mavinic e Beckie (2007) \\
\hline $\mathrm{HPO}_{4}^{2-}+\mathrm{H}^{+} \leftrightarrow \mathrm{H}_{2} \mathrm{PO}_{4}^{-}$ & 7,20 & $\begin{array}{l}\text { Webb e Ho (1991), Bhuyian, Mavinic e Beckie (2007), } \\
\text { Bouropoulos e Koutsoukos (2000), Babic-Ivancic et al. } \\
\text { (2002), Doyle e Parsons (2002), Ronteltap, Maurer e Gujer } \\
\text { (2007), Hanhoun et al. (2011), Rahaman et al. (2014) }\end{array}$ \\
\hline $\mathrm{SO}_{4}^{2-}+\mathrm{H}^{+} \leftrightarrow \mathrm{HSO}_{4}^{-}$ & 1,99 & Babic-Ivancic et al. (2002) \\
\hline $\mathrm{Ca}(\mathrm{OH})_{2} \leftrightarrow \mathrm{Ca}^{2+}+2 \mathrm{OH}^{-}$ & $-5,2$ & Musvoto et al. (2000), Mehta e Batstone (2013) \\
\hline $\mathrm{Mg}\left(\mathrm{OH}_{2}\right) \cdot 6 \mathrm{H}_{2} \mathrm{O} \leftrightarrow 6 \mathrm{H}_{2} \mathrm{O}+\mathrm{Mg}^{2+}+2 \mathrm{OH}^{-}$ & $-10,9\left(K_{P S}\right)$ & Mehta e Batstone (2013) \\
\hline $\mathrm{Mg}(\mathrm{OH})_{2} \leftrightarrow \mathrm{Mg}^{2+}+2 \mathrm{OH}^{-}$ & $-11,16\left(K_{P S}\right)$ & Musvoto et al. (2000), Çelen et al. (2007) \\
\hline $\mathrm{Mg}^{2+}+\mathrm{OH}^{-} \leftrightarrow \mathrm{MgOH}^{+}$ & 2,6 & $\begin{array}{l}\text { Bouropoulos e Koutsoukos (2000), Doyle e Parsons } \\
\text { (2002), Hanhoun et al. (2011), Rahaman et al. (2014) }\end{array}$ \\
\hline $\mathrm{Mg}^{2+}+\mathrm{H}_{2} \mathrm{O} \leftrightarrow \mathrm{MgOH}^{+}+\mathrm{H}^{+}$ & $-11,44$ & Webb e Ho (1991), Bhuyian, Mavinic e Beckie (2007) \\
\hline $\mathrm{Ca}_{10}\left(\mathrm{PO}_{4}\right)_{6}(\mathrm{OH})_{2} \leftrightarrow 10 \mathrm{Ca}^{2+}+6 \mathrm{PO}_{4}^{3-}+2 \mathrm{OH}^{-}$ & $-114\left(K_{P S}\right)$ & Musvoto et al. (2000) \\
\hline $\mathrm{Ca}_{3}\left(\mathrm{PO}_{4}\right)_{2} \cdot \mathrm{xH}_{2} \mathrm{O} \leftrightarrow 3 \mathrm{Ca}^{2+}+2 \mathrm{PO}_{4}^{3}+\mathrm{xH}_{2} \mathrm{O}$ & $-31,45\left(K_{P S}\right)$ & Musvoto et al. (2000) \\
\hline $\mathrm{Ca}_{3}\left(\mathrm{PO}_{4}\right)_{2} \leftrightarrow 3 \mathrm{Ca}^{2+}+2 \mathrm{PO}_{4}^{3-}$ & $-32,7\left(K_{P S}\right)$ & Musvoto et al. (2000), Mehta e Batstone (2013) \\
\hline $\mathrm{Ca}_{8}\left(\mathrm{HPO}_{4}\right)_{2}\left(\mathrm{PO}_{4}\right)_{4} 5 \mathrm{H}_{2} \mathrm{O} \leftrightarrow 8 \mathrm{Ca}^{2+}+2 \mathrm{HPO}_{4}^{2-}+4 \mathrm{PO}_{4}^{3-}+5 \mathrm{H}_{2} \mathrm{O}$ & $-94,16\left(K_{P S}\right)$ & Musvoto et al. (2000) \\
\hline $\mathrm{CaHPO}_{4} \leftrightarrow \mathrm{Ca}^{2+}+\mathrm{HPO}_{4}^{2-}$ & $-2,7\left(K_{P S}\right)$ & Musvoto et al. (2000) \\
\hline $\mathrm{CaHPO}_{4} \cdot 2 \mathrm{H}_{2} \mathrm{O} \leftrightarrow \mathrm{Ca}^{2+}+\mathrm{HPO}_{4}^{2-}+2 \mathrm{H}_{2} \mathrm{O}$ & $-6,6\left(K_{P S}\right)$ & Musvoto et al. (2000), Mehta e Batstone (2013) \\
\hline $\mathrm{Ca}_{5}\left(\mathrm{PO}_{4}\right)_{3}(\mathrm{OH}) \leftrightarrow 5 \mathrm{Ca}^{2+}+3 \mathrm{PO}_{4}^{3-}+\mathrm{OH}^{-}$ & $-57,8\left(K_{P S}\right)$ & Muster et al. (2013) \\
\hline $\mathrm{H}_{2} \mathrm{PO}_{4}^{-}+\mathrm{Mg}^{2+} \leftrightarrow \mathrm{MgH}_{2} \mathrm{PO}_{4}^{+}$ & 0,45 & $\begin{array}{l}\text { Bhuyian, Mavinic e Beckie (2007), Bouropoulos e } \\
\text { Koutsoukos (2000), Babic-Ivancic et al. (2002), Doyle e } \\
\text { Parsons (2002), Hanhoun et al. (2011), Rahaman et al. (2014) }\end{array}$ \\
\hline $\mathrm{H}_{2} \mathrm{PO}_{4}^{-}+\mathrm{Mg}^{2+} \leftrightarrow \mathrm{MgPO}_{4}^{-}+2 \mathrm{H}^{+}$ & $-12,96$ & Ronteltap, Maurer e Gujer (2007) \\
\hline $\mathrm{H}_{2} \mathrm{PO}_{4}^{-}+\mathrm{Mg}^{2+} \leftrightarrow \mathrm{MgHPO}_{4}+\mathrm{H}^{+}$ & $-4,3$ & Ronteltap, Maurer e Gujer (2007) \\
\hline $\mathrm{HPO}_{4}^{2-}+\mathrm{Mg}^{2+} \leftrightarrow \mathrm{MgHPO}_{4}$ & 2,43 & $\begin{array}{l}\text { Babic-Ivancic et al. (2002), Doyle e Parsons (2002), Hanhoun } \\
\text { et al. (2011), Rahaman et al. (2O14), Bhuyian, Mavinic e Beckie } \\
\text { (2007), Bouropoulos e Koutsoukos (2000) }\end{array}$ \\
\hline $\mathrm{PO}_{4}^{3-}+\mathrm{Mg}^{2+} \leftrightarrow \mathrm{MgPO}_{4}^{-}$ & 4,80 & $\begin{array}{l}\text { Bhuyian, Mavinic e Beckie (2007), Bouropoulos e } \\
\text { Koutsoukos (2000), Babic-Ivancic et al. (2002), } \\
\text { Doyle e Parsons (2002), Hanhoun et al. (2011) }\end{array}$ \\
\hline $\mathrm{Mg}_{3} \mathrm{HPO}_{4} \cdot 3 \mathrm{H}_{2} \mathrm{O} \leftrightarrow 3 \mathrm{H}_{2} \mathrm{O}+\mathrm{HPO}_{4}^{2-}+\mathrm{Mg}^{2+}$ & $-5,8\left(K_{P S}\right)$ & Musvoto et al. (2000), Çelen et al. (2007) \\
\hline
\end{tabular}


Tabela 2 - Continuação.

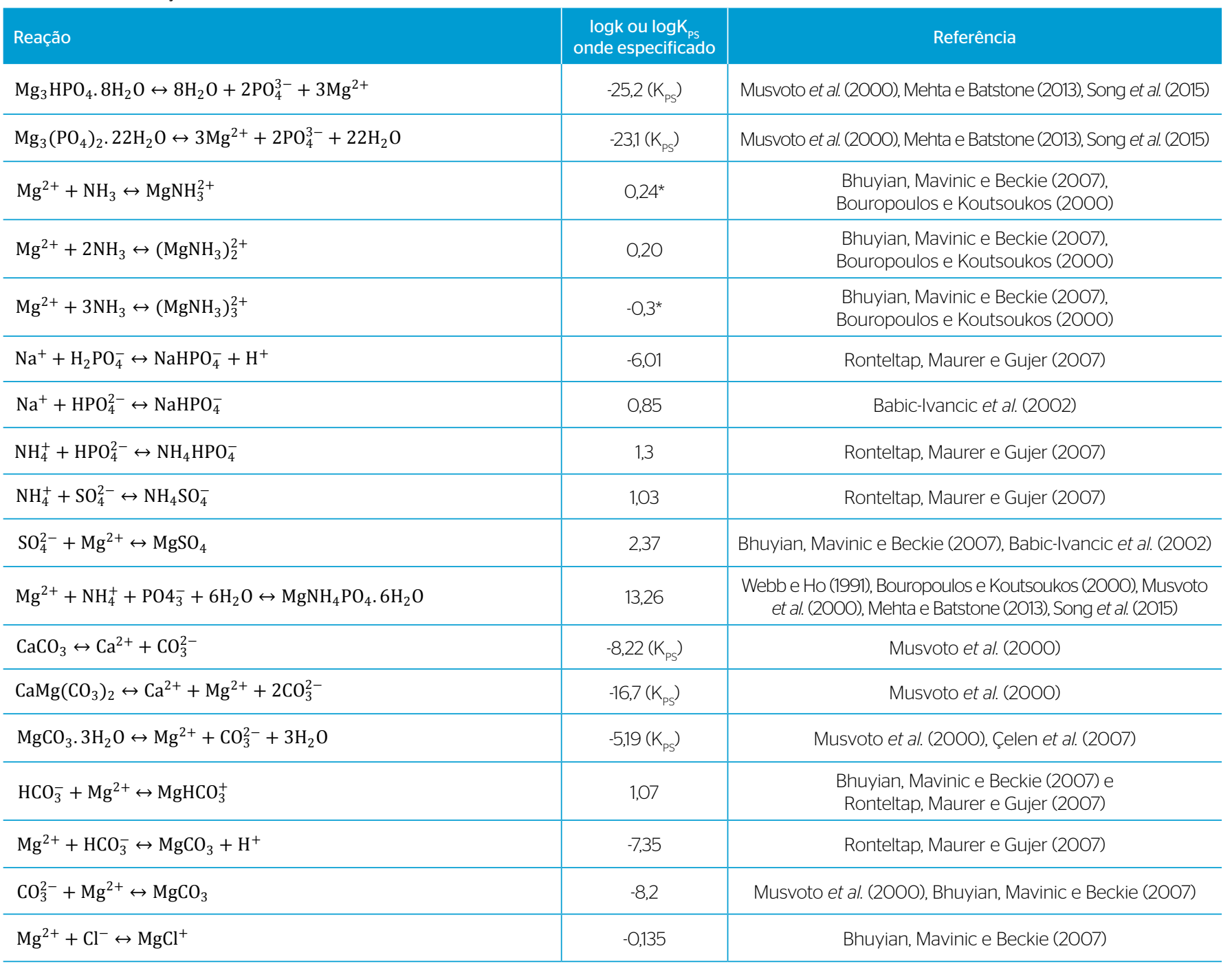

*I $=0,2 \mathrm{M} \mathrm{em} \mathrm{NH}_{4} \mathrm{NO}_{3}$.

Entretanto, Bhuyian, Mavinic e Beckie (2007) e Hanhoun et al. (2011) obtiveram diferenças significativas entre os valores dos produtos de solubilidade da estruvita, calculados com as constantes das reações corrigidas pela equação de Van’t Hoff, e pelas Equações 2 a 5, que consideram a capacidade calorífica dependente da temperatura.

$\ln \left(\frac{\mathrm{k}_{\mathrm{T}}}{\mathrm{k}_{298}}\right)=\frac{\Delta \mathrm{H}_{298 \mathrm{~K}}^{0}}{\mathrm{R}} \cdot\left(\frac{1}{298}-\frac{1}{\mathrm{~T}}\right)$

$\Delta \mathrm{G}_{\mathrm{T}}=-\mathrm{R} \cdot \mathrm{T} \cdot \ln _{\mathrm{T}}$

$\Delta \mathrm{G}_{\mathrm{T}}=\Delta \mathrm{H}_{\mathrm{T}}-\mathrm{T} \cdot \Delta \mathrm{S}_{\mathrm{T}}$

$\Delta \mathrm{H}_{\mathrm{T}}=\Delta \mathrm{H}_{298 \mathrm{~K}}^{0}+\int_{298 \mathrm{~K}}^{\mathrm{T}} \Delta \mathrm{C}_{\mathrm{p}} \mathrm{dT}$
$\Delta \mathrm{S}_{\mathrm{T}}=\Delta \mathrm{S}_{298 \mathrm{~K}}^{0}+\int_{298 \mathrm{~K}}^{\mathrm{T}} \frac{\Delta \mathrm{C}_{\mathrm{p}}}{\mathrm{T}} \cdot \mathrm{dT}$

Em que:

$k_{T}$ : constante de equilíbrio na temperatura $\mathrm{T}$ (adimensional);

$k_{298}$ : constante de equilíbrio a $25^{\circ} \mathrm{C}$ (adimensional);

$\Delta H_{298 K}^{0}$ : calor de (ou variação de entalpia na) formação (transformação) de uma substância pura, em temperatura de $25^{\circ} \mathrm{C}(298 \mathrm{~K})$ e pressão de $1 \mathrm{~atm}\left(\mathrm{~J} \cdot \mathrm{mol}^{-1}\right)$;

$R$ : constante universal dos gases $\left(\mathrm{J} \cdot \mathrm{mol}^{-1} \cdot \mathrm{K}^{-1}\right)$;

$T$ : temperatura $(\mathrm{K})$;

$\Delta G_{T}$ : energia livre de Gibbs da reação na temperatura T (J.mol-1);

$\Delta H_{T}$ : variação do calor de formação (transformação) de uma substância em uma temperatura T (J.mol $\left.{ }^{-1}\right)$;

$\Delta S_{T}$ : variação da entropia na formação (transformação) de uma substância em uma temperatura T $\left(\mathrm{J} \cdot \mathrm{K}^{-1} \cdot \mathrm{mol}^{-1}\right)$; 
$\Delta S_{298 K}^{0}$ : variação de entropia na formação (transformação) de uma substância pura, em temperatura de $25^{\circ} \mathrm{C}(298 \mathrm{~K})$ e pressão de $1 \mathrm{~atm}$ $\left(\mathrm{J} \cdot \mathrm{K}^{-1} \cdot \mathrm{mol}^{-1}\right)$;

$\Delta C_{p}$ : é a variação capacidade calorífica $\left(\mathrm{J} \cdot \mathrm{mol}^{-1}\right)$.

A intensidade iônica do meio altera os valores de $\mathrm{k}$, que podem ser corrigidos usando diferentes equações, sendo a de Davies (Equação 6) a mais empregada por aqueles que estudaram a formação da estruvita. Entretanto, é importante ressaltar que ela só é válida para I menor do que 0,5 mol. $\mathrm{L}^{-1}$ :

$\log \mathrm{k}=-\mathrm{A} \cdot \mathrm{i}^{2} \cdot\left(\frac{\sqrt{\mathrm{I}}}{1+\sqrt{\mathrm{I}}}-0,3 \cdot \mathrm{I}\right)$

Na qual:

$\mathrm{k}=$ constante de equilíbrio num meio com intensidade iônica I (adimensional);

$\mathrm{i}=$ carga do íon (adimensional);

$\mathrm{I}=$ intensidade iônica do meio $\left(\mathrm{mol} \cdot \mathrm{L}^{-1}\right)$.

Segundo Bhuyian, Mavinic e Beckie (2009), para sobrenadante de digestor anaeróbio de lodo de sistema EBPR, I pode ser calculada em função da condutividade em $25^{\circ} \mathrm{C}\left(\mathrm{C}_{25}\right)$, expressa em $\mu \mathrm{S} . \mathrm{cm}^{-1}$, pela Equação 7:

$\mathrm{I}=7,22 \cdot 10^{-6} \cdot C_{25}$

$\mathrm{A}=$ constante de Debye-Hückel (adimensional), que pode ser determinada a partir da Equação 8:

$A=1,82 \times 10^{6} .(\varepsilon . T)^{-3 / 2}$

Em que:

$\varepsilon=$ constante dielétrica da água (adimensional), que pode ser calculada com a Equação 9, na qual T é a temperatura em K.

$\varepsilon=308,67 . \mathrm{e}^{-0,0045976 . \mathrm{T}}$

A concentração total de cada elemento, representada como $\mathrm{C}_{\text {TPO4, }}$ $\mathrm{C}_{\mathrm{TNH} 4}$ e $\mathrm{C}_{\mathrm{TMg}}$, para o fósforo, o nitrogênio e o magnésio, respectivamente, corresponde à somatória de todas as espécies presentes na solução. Assim, considerando que as espécies formadoras da estruvita são $\mathrm{Mg}^{2+}, \mathrm{NH}_{4}^{+}$e $\mathrm{PO}_{4}^{3-}$, suas frações reativas $\alpha$ são calculadas de acordo com as Equações 10 a 12, respectivamente.

$\alpha_{\mathrm{Mg}^{2+}}=\frac{\left[\mathrm{Mg}^{2+}\right]}{\mathrm{C}_{\mathrm{TMg}}}$

$\alpha_{\mathrm{NH}_{4}}+=\frac{\left[\mathrm{NH}_{4}{ }^{+}\right]}{\mathrm{C}_{\mathrm{TNH}_{4}}}$
$\alpha_{\mathrm{PO}_{4}{ }^{3-}}=\frac{\left[\mathrm{PO}_{4}{ }^{3-}\right]}{\mathrm{C}_{\mathrm{TPO}}{ }_{4}}$

\section{Solubilidade da estruvita}

Como outras reações de precipitação, a da estruvita é controlada por sua solubilidade (AAGE et al., 1997; BURNS \& FINLAYSON, 1982; TAYLOR et al., 1963; WEBB; HO, 1991). Diferentes pesquisadores têm utilizado diferentes tipos de produtos de solubilidade para verificar seu potencial de precipitação. A seguir, são apresentados os conceitos de tais produtos, bem como a viabilidade de seu uso.

Considerando a reação de dissolução da estruvita (Reação 3),

$\mathrm{MgNH}_{4} \mathrm{PO}_{4} \cdot 6 \mathrm{H}_{2} \mathrm{O}(\mathrm{s}) \rightarrow \mathrm{Mg}^{2+}+\mathrm{NH}_{4}^{+}+\mathrm{PO}_{4}^{3-}+6 \mathrm{H}_{2} \mathrm{O}$

Sua constante do produto de solubilidade termodinâmica $\left(\mathrm{K}_{\mathrm{PS}}\right)$ é expressa pela Equação 13:

$K_{P S}=\left[\mathrm{Mg}^{2+}\right] \cdot\left[\mathrm{NH}_{4}^{+}\right] \cdot\left[\mathrm{PO}_{4}^{3-}\right]$

A Equação 13 é de difícil aplicação para o esgoto sanitário ou águas residuárias industriais, devido à presença e à influência de muitas outras espécies dissolvidas além dos componentes da estruvita. Ela é válida apenas em situações de diluição infinita, isto é, quando o efeito das condições do meio ( $\mathrm{pH}$, intensidade iônica etc.) pode ser desprezado. Segundo Mullin (2001), isso ocorre quando as concentrações das espécies iônicas são menores do que $10^{-3} \mathrm{~mol} . \mathrm{L}^{-1}$. Uma maneira mais adequada de se avaliar a solubilidade da estruvita em esgoto/águas residuárias é pela constante do produto de solubilidade de atividade, $\mathrm{K}_{\mathrm{so}}$, que considera as atividades $\left(\mathrm{a}_{\mathrm{i}}\right)$ das espécies iônicas que a formam. Ela pode ser determinada pela Equação 14:

$\left.K_{S o}=\left(a_{M g^{2+}}\right) \cdot\left(a_{N H_{4}^{+}}\right) \cdot\left(a_{P O_{4}^{3}}\right)=\left\{\left(\gamma_{M g^{2+}}\right) \cdot\left[M g^{2+}\right]\right)\right\} \cdot\left\{\left(\gamma_{N H_{4}^{+}}\right) \cdot\left[N_{4}^{+}\right]\right\} \cdot\left\{\left(\gamma_{P O_{4}^{3}}\right) \cdot\left[\mathrm{PO}_{4}^{3-}\right]\right\}$

Em que:

$a_{i}=a$ atividade do i-ésimo íon em solução, definido como $a_{i}=\gamma_{i} \cdot\left[C_{i}\right]$;

$\gamma_{\mathrm{i}}=\mathrm{o}$ coeficiente de atividade da í-ésima espécie iônica;

$\left[\mathrm{C}_{\mathrm{i}}\right]=$ é sua respectiva concentração.

Entretanto, dependendo do número de íons presentes na solução, o cálculo de $\mathrm{K}_{\mathrm{So}}$ pode ser bastante trabalhoso. Para facilitar a avaliação do estado de saturação de uma solução, Schwarzenbach (1957) definiu o termo "produto de solubilidade condicional $\left(\mathrm{P}_{\mathrm{s}}\right)$ " como o produto das concentrações totais de cada elemento presente na solução. Para o caso da estruvita, o Ps é calculado pela Equação 15:

$P_{s}=C_{T M g} \cdot C_{T N H_{4}} \cdot C_{T_{P O}}$ 
Das Equações 13, 14 e 15, obtém-se a Equação 16:

$$
\mathrm{P}_{\mathrm{S}}=\frac{\mathrm{K}_{\mathrm{PS}}}{\left(\alpha_{\mathrm{Mg}^{2+}}\right) \cdot\left(\alpha_{\mathrm{NH}_{4}^{+}}\right) \cdot\left(\alpha_{\mathrm{PO}_{4}^{3-}}\right)}=\frac{\mathrm{K}_{\mathrm{S} 0}}{\left(\alpha_{\mathrm{Mg}^{2+}}\right) \cdot\left(\gamma_{\mathrm{Mg}^{2+}}\right) \cdot\left(\alpha_{\mathrm{NH}_{4}^{+}}\right) \cdot\left(\gamma_{\mathrm{NH}_{4}^{+}}\right) \cdot\left(\alpha_{\mathrm{PO}_{4}^{3-}}\right) \cdot\left(\gamma_{\mathrm{PO}_{4}^{3-}}\right)}
$$

Três situações podem ocorrer:

- se $\mathrm{P}_{\mathrm{S}}>\mathrm{K}_{\mathrm{S} 0}$, a solução está supersaturada, indicando que a concentração do soluto excede o valor de equilíbrio substancialmente, causando nucleação espontânea. A cristalização é rápida e abundante, sem a necessidade de semeadura;

- $\quad$ se $\mathrm{P}_{\mathrm{S}}=\mathrm{K}_{\mathrm{S} 0}$, a solução está saturada em uma região denominada de metaestável. A nucleação espontânea não ocorre nessa região, mas o crescimento cristalino pode ser induzido por semeadura;

- se $\mathrm{P}_{\mathrm{S}}<\mathrm{K}_{\mathrm{s} 0}$, a solução não está saturada, indicando que cristais presentes serão dissolvidos quando adicionados na solução. A cristalização é impossível.

A Tabela 3 mostra alguns valores de $\mathrm{pK}_{\mathrm{S} 0}$ para a estruvita, reportados por diferentes pesquisadores. As diferenças entre os valores podem ser justificadas pelas seguintes razões (ANDRADE; SCHUILING, 2001; STUMM; MORGAN, 1996):

- a complexidade dos equilíbrios heterogêneos é maior do que a dos homogêneos;

- o efeito da força iônica não foi considerado nos cálculos;

- espécies químicas diferentes foram selecionadas para a realização dos cálculos;

- não foram considerados os complexos que afetam os equilíbrios químicos.

Nota-se pela Tabela 3 que os valores das constantes são mais baixos para águas residuárias reais do que para soluções preparadas em laboratório.

Como as concentrações das espécies químicas variam com o $\mathrm{pH}$, com a intensidade iônica e com a temperatura do meio, o $\mathrm{K}_{\mathrm{S} 0}$ também sofrerá a influência destas variáveis. Aage et al. (1997) mostraram que ele aumentava continuamente de 10 a $50^{\circ} \mathrm{C}$, a partir do qual começava a decrescer até $65^{\circ} \mathrm{C}$. Bhuyian, Mavinic e Beckie (2007) e Hanhoun et al. (2011) também observaram esse mesmo comportamento, só que o valor máximo se deu em $30^{\circ} \mathrm{C}$. Já Rahaman et al. (2006) notaram que o $\mathrm{K}_{\mathrm{s} 0}$ não era significativamente influenciado pela temperatura no intervalo de $\mathrm{pH}$ entre 5,5 e 7,0; acima de 7,0, ele aumentava quando a temperatura passava de 10 para $20^{\circ} \mathrm{C}$.

A maioria dos pesquisadores concluiu que a solubilidade da estruvita é mínima em uma faixa de $\mathrm{pH}$ de 8,0 a 11,0 (NELSON; MIKKELSEN; HESTERBERG, 2003; WANG et al., 2005; ALI, 2007; YIGIT; MAZLUM, 2007; BHUYIAN; MAVINIC; BECKIE, 2007; HANHOUN et al., 2011; CERRILLO et al., 2015; REN et al., 2015) e que ela aumenta com a intensidade iônica, devido às interações eletrostáticas, que reduzem as atividades das espécies iônicas (RONTELTAP; MAURER; GUJER, 2007; OHLINGER; YOUNG; SCHROEDER, 1998; TAO; FATTAH; HUCHZERMEIER, 2016).

\section{Supersaturação}

Há diferentes formas de se expressar a supersaturação e, por essa razão, deve-se tomar cuidado na aplicação das fórmulas ou na comparação dos resultados obtidos pelos diversos pesquisadores. Segundo Mullin (2001), considerando que a força motriz da cristalização seja a diferença de concentração de um sal $(\mathrm{C})$ na solução em relação à solubilidade molar $\left(C^{*}\right)$, em uma dada temperatura, define-se razão de supersaturação (S) a relação entre as duas (Equação 17):

$\mathrm{S}=\frac{\mathrm{C}}{\mathrm{C}^{*}}$

Considerando a Reação 3, $\mathrm{C}^{*}=\left[\mathrm{Mg}^{2+}\right]=\left[\mathrm{PO}_{4}^{3-}\right]=\left[\mathrm{NH}_{4}^{+}\right]$. Mas, de acordo com a Equação 13: $\mathrm{K}_{\mathrm{PS}}=\left[\mathrm{Mg}^{2+}\right] .\left[\mathrm{PO}_{4}^{3-}\right] .\left[\mathrm{NH}_{4}^{+}\right]$, então $C^{*}=\left(K_{\mathrm{PS}}\right)^{\frac{1}{3}}$

A supersaturação relativa é dada pela Equação 18:

$\sigma=\frac{\Delta C}{C^{*}}=S-1$

No caso de soluto não solvatado e solução binária, a força motriz é expressa pela diferença entre os potenciais químicos de uma dada substância na solução supersaturada $\left(\mu_{1}\right)$ e no equilíbrio $\left(\mu_{2}\right)$ (Equação 19):

$\Delta \mu=\mu_{1-} \mu_{2}$

Entretanto, $\mu=\mu_{0}+$ RTlna

Em que:

$\mu_{0}=$ potencial químico no estado padrão;

$\mathrm{a}=$ atividade

Assim, tem-se a Equação 20:

$\frac{\Delta \mu}{\mathrm{RT}}=\ln \frac{\mathrm{a}}{\mathrm{a}^{*}}=\ln \mathrm{S}$

Para soluções eletrolíticas, em vez da atividade, usa-se a atividade iônica, sendo $\mathrm{a}=\mathrm{a}_{\mathrm{i}}^{\mathrm{z}}$, na qual $\mathrm{z}=\mathrm{z}_{+}+\mathrm{z}_{-}=$número de mol de íons por mol de eletrólito. Então, a Equação 20 torna-se a Equação 21:

$\frac{\Delta \mu}{\mathrm{RT}}=\mathrm{z} \ln \frac{\mathrm{a}_{\mathrm{i}}}{\mathrm{a}_{\mathrm{i}}^{*}}=\mathrm{z} \ln \mathrm{S}_{\mathrm{a}}$

$\mathrm{S}_{\mathrm{a}}=$ razão de supersaturação baseada em atividade.

Para estruvita (Equação 22):

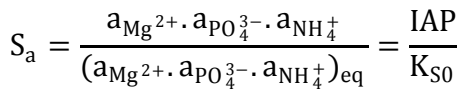


Na qual:

IAP $=$ produto de atividade iônica.

Para soluções aquosas de eletrólitos fracamente solúveis, $\mathrm{S}_{\mathrm{a}}$ pode ser calculada pela Equação 23:

$\mathrm{S}_{\mathrm{a}}=\left(\frac{\mathrm{IAP}}{\mathrm{K}_{\mathrm{S} 0}}\right)^{\frac{1}{\mathrm{z}}}$

Sendo $\mathrm{z}=3$ para a estruvita.

\section{Modelagem matemática}

Como visto, muitas variáveis (concentrações de nitrogênio, de fósforo e de magnésio; relação Mg:N:P; pH; temperatura; intensidade iônica; presença de impurezas; razão de supersaturação) influenciam na formação da estruvita. Diversas pesquisas são realizadas sem considerar o efeito combinado de todas essas variáveis e sua alteração conforme o tempo de reação. Por tentativa e erro, varia-se o pH e a razão $\mathrm{Mg}: \mathrm{P}: \mathrm{N}$ e são analisados os difratogramas de raios $\mathrm{X}$ dos sólidos produzidos e as remoções de P, N e Mg. Entretanto, os valores ótimos encontrados são válidos apenas para o caso estudado e a difração de raios $\mathrm{X}$ não serve para a verificação da pureza da estruvita, como demonstrado por Hao et al. (2008), que sugerem, para essa determinação, a análise elementar dos precipitados dissolvidos em ácido. Atualmente, são utilizados programas computacionais para:

- avaliar o potencial de formação de estruvita (ALI, 2007; LEE et al., 2013);

- averiguar os fatores que influenciam essa formação (BUCHANAN; MOTE; ROBINSON, 1994; ÇELEN et al., 2007; ZHANG et al., 2011);

- quantificar a estruvita formada (DOYLE et al., 2000);

- prever as fases que cristalizam e precipitam (LEE et al., 2013; ZHOU; WU, 2012).

Os modelos termodinâmicos mais empregados são: "STRUVITE” (DOYLE et al., 2000), "Visual MINTEQ" (ALI; SCHNEIDER; HUDSON, 2003; ÇELEN et al., 2007; YANG et al., 2011; ZHOU; WU, 2012; CRUTCHIK; SÁNCHEZ; GARRIDO, 2013; LEE et al., 2013), "MINTEQA2" (BUCHANAN; MOTE; ROBINSON, 1994), "PHREEQC" (ALI, 2007; BHUYIAN; MAVINIC; BECKIE, 2007; ZHANG et al., 2011; MUSTER et al., 2013; SONG et al., 2015),

Tabela 3 - Valores da constante do produto de solubilidade de atividade $\left(\mathrm{pK}_{\mathrm{so}}\right)$ da estruvita obtidos por diferentes pesquisadores.

\begin{tabular}{|c|c|c|}
\hline $\mathrm{pK}_{\mathrm{so}}$ & Condições em KSo foi determinada & Referência \\
\hline $12,76 \pm 0,56$ & $\begin{array}{l}\text { Água residuária de suinocultura, }\left[\mathrm{P}_{\text {solivel }}\right]=65 \mathrm{mg} \cdot \mathrm{L}^{-1},\left[\mathrm{~N}-\mathrm{NH}_{3}\right]=1830 \mathrm{mg} \cdot \mathrm{L}^{-1} \text {, } \\
{[\mathrm{Mg}]=170 \mathrm{mg} \cdot \mathrm{L}^{-1}, \mathrm{pH} \text { entre } 6,8 \text { e } 8,5 \text {, temperatura }=25^{\circ} \mathrm{C}}\end{array}$ & Webb e Ho (1991) \\
\hline 14 & $\begin{array}{l}\text { Dissolução de } 40 \text { mg de estruvita marcada com o radioisótopo }{ }^{32} \mathrm{P} \text { em } 20 \mathrm{~mL} \text { de } \\
\text { água redestilada e soluções diluídas de } \mathrm{NaCl} \text { 0,030, 0045, 0,06 M, temperatura = } 25^{\circ} \mathrm{C}\end{array}$ & Aage et al. (1997) \\
\hline $13,26 \pm 0,04$ & $\begin{array}{l}\text { Produção de estruvita em laboratório. Dissolução desta estruvita e de estruvita coletada em } \\
\text { sobrenadante de digestor de sistema EBPR em água desionizada tamponada, temperatura }=25 \pm 0,5^{\circ} \mathrm{C}\end{array}$ & $\begin{array}{l}\text { Ohlinger, Young e } \\
\text { Schroeder (1998) }\end{array}$ \\
\hline $13,36 \pm 0,28$ & $\begin{array}{l}\text { Agua sintética; }[\mathrm{Pt}]=310 \text { a } 1.860 \mathrm{mg} \cdot \mathrm{L}^{-1} ;\left[\mathrm{N}-\mathrm{NH}_{3}\right]=410 \text { a } 1.568 \mathrm{mg} . \mathrm{L}^{1} \text {; } \\
{[\mathrm{Mg}]=240 \text { a } 552 \mathrm{mg} \cdot \mathrm{L}^{-1}=\text { temperatura } 25^{\circ} \mathrm{C} ; \mathrm{pH}^{3}=7,4 ;[\mathrm{NaCl}]=0,15 \mathrm{~mol} \cdot \mathrm{L}^{-1}}\end{array}$ & Babic-Ivancic et al. (2002) \\
\hline $14,10 \pm 0,16$ & Dissolução de cristais de estruvita em água destilada; $\mathrm{pH}=7,01$ a 9,52; temperatura de $20^{\circ} \mathrm{C}$ & \multirow{4}{*}{ Rahaman et al. (2006) } \\
\hline $13,55 \pm 0,54$ & $\begin{array}{l}\text { Dissolução de cristais de estruvita no sobrenadante do digestor } \\
\text { da ETE Annacis Is/and; } \mathrm{pH}=5,62 \text { a 8,77; temperatura de } 20^{\circ} \mathrm{C}\end{array}$ & \\
\hline $13,53 \pm 0,45$ & $\begin{array}{l}\text { Dissolução de cristais de estruvita no sobrenadante do digestor } \\
\text { da ETE Lulu Island; } \mathrm{pH}=5,89 \text { a 9,02; temperatura de } 20^{\circ} \mathrm{C}\end{array}$ & \\
\hline $13,45 \pm 0,37$ & $\begin{array}{l}\text { Dissolução de cristais de estruvita no sobrenadante do digestor } \\
\text { da ETE Penticton; } \mathrm{pH}=6,45 \text { a 8,97; temperatura de } 20^{\circ} \mathrm{C}\end{array}$ & \\
\hline $13,69 \pm 0,02$ & $\begin{array}{l}\text { Dissolução de cristais de estruvita em água desionizada contendo } \\
\text { solução de } \mathrm{NaCl} \text { 0,01; 0,02; 0,04 e 0,06 M; } \mathrm{pH}=7,4 \text {; temperatura de } 2 \mathrm{O}^{\circ} \mathrm{C}\end{array}$ & \multirow{2}{*}{$\begin{array}{l}\text { Bhuyian, Mavinic e } \\
\text { Beckie (2007) }\end{array}$} \\
\hline $13,36 \pm 0,07$ & $\begin{array}{l}\text { Dissolução de cristais de estruvita em água desionizada contendo } \\
\text { solução de } \mathrm{NaCl} 0,01 ; 0,02 ; 0,04 \text { e 0,06 M; } \mathrm{pH}=7,4 \text {; temperatura de } 25^{\circ} \mathrm{C}\end{array}$ & \\
\hline $13,26 \pm 0,06$ & 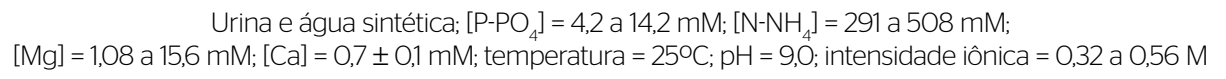 & $\begin{array}{l}\text { Ronteltap, Maurer e } \\
\quad \text { Gujer (2007) }\end{array}$ \\
\hline 11 a 12 & $\begin{array}{l}\text { Efluente de digestor anaeróbio tratando água residuária de indústria de melaço, } \\
\left.\left.\left[\mathrm{NH}_{4}\right]=1400 \mathrm{mg} \mathrm{L}^{-1} ; \mathrm{Mg}^{2+}\right]=21 \mathrm{mg} . \mathrm{L}^{-1} ; \mathrm{PO}_{4}^{3-}\right]=24 \mathrm{mg} \mathrm{L}^{-1} ;\left[\mathrm{Ca}^{2+}\right]=21 \mathrm{mg} \cdot \mathrm{L}^{-1} ;\left[\mathrm{K}^{+}\right]=2.150 \mathrm{mg} \cdot \mathrm{L}^{-1} ; \\
\text { sólidos dissolvidos totais }=7.600 \mathrm{mg} \cdot \mathrm{L}^{-1} ; \mathrm{PH}=7,9 \text {, temperatura }=37 \mathrm{C}^{\circ} \mathrm{C}\end{array}$ & Türker e Çelen (2010) \\
\hline $13,17 \pm 0,01$ & $\begin{array}{l}\text { Água sintética; }\left[\mathrm{PO}_{4}\right]=4 \mathrm{mM} ;[\mathrm{Mg}]=4 \mathrm{mM} \text { e }\left[\mathrm{NH}_{4}\right]=4 \mathrm{mM} \\
\text { temperatura = 250 } \mathrm{C} ; \mathrm{pH}=8,5 ; 9,0 ; 9,5 \text { e 10,0; intensidade iônica = 0,0159 M }\end{array}$ & Hanhoun et al. (2011) \\
\hline 14,0 & $\begin{array}{l}\text { Agua sintética; }\left[\mathrm{PO}_{4}^{3-}\right]=2 \mathrm{mM} ;\left[\mathrm{NH}_{4}^{+}\right]=2,2 \mathrm{mM} ;\left[\mathrm{Mg}^{2+}\right]=6,0 \mathrm{mM} \text {; } \\
\text { temperatura } 25^{\circ} \mathrm{C} ; \mathrm{pH}=8,5 ; 3 \mathrm{~g} \cdot \mathrm{L}^{-1} \text { de estruvita adicionada }\end{array}$ & Crutchik e Garrido (2016) \\
\hline
\end{tabular}


gPROMS (ALI, 2007), ENGINEERING EQUATION SOLVER (GALBRAITH; SCHNEIDER, 2009) e "MINEQL+" (WANG et al., 2005). No entanto, raramente os resultados são validados em escala real. Doyle et al. (2000) fizeram essa verificação e obtiveram coeficiente de determinação $\left(\mathrm{r}^{2}\right)$ de 0,93 ao compararem as massas de estruvita produzidas na escala de laboratório, em diferentes valores de $\mathrm{pH}$, com os resultados previstos pelo modelo STRUVITE. Para a escala real, os valores de $\mathrm{r}^{2}$ foram de 0,75 e 0,82 , respectivamente, quando as massas foram calculadas por meio das remoções de magnésio e de fósforo. Mesmo em escala de laboratório, Muster et al. (2013), que usaram o PHREEQC, e Nelson, Mikkelsen e Hesterberg (2003), que empregaram o MINTEQA2, verificaram diferenças significativas entre as previsões feitas pelos modelos e os resultados obtidos experimentalmente. Recentemente, Lee, Kumar e Jeon (2016) desenvolveram um modelo dinâmico, que incorporou o declínio do $\mathrm{pH}$ ao longo da reação de produção de estruvita, a volatilização da amônia e a interferência do cálcio. Os pesquisadores calibraram o modelo com experimentos em escala de laboratório e concluíram que ele previa muito bem a formação da estruvita, mas não conseguia prognosticar outros precipitados que eram formados.

Portanto, a comunidade técnica e científica deve fazer um esforço para o desenvolvimento de programas robustos e validá-los em ETEs reais, como as existentes no Japão, Holanda, Alemanha, Canadá, Inglaterra, Estados Unidos, Austrália e Itália, de modo a desenvolver projetos mais adequados e melhorar a operação.

\section{Tipos de nucleação}

\section{Nucleação primária homogênea}

Na nucleação primária homogênea, ocorrem colisões sequenciais entre os íons presentes na solução supersaturada, resultando na formação de pequenos clusters (agregados de átomos ou moléculas), que crescem. Os que atingem um tamanho igual ao crítico são chamados de núcleos críticos ou clusters críticos; os maiores, simplesmente de núcleos; e os menores, de embriões. Os núcleos diminuirão suas energias livres por meio do crescimento, pela incorporação de átomos ou moléculas. Os embriões tenderão a dissolver-se para reduzir sua energia livre; os átomos ou moléculas migrarão para fora da partícula, retornando ao líquido. Os núcleos críticos poderão crescer ou dissolver-se, pois ambos os processos diminuem suas energias livres.

A seguir, são apresentadas as equações fundamentais da nucleação homogênea, acompanhadas dos valores dos parâmetros obtidos para a estruvita por diferentes pesquisadores. Considerou-se, por simplificação, uma partícula esférica com raio "r".

A energia total necessária à formação de um $\operatorname{cluster}(\Delta \mathrm{G})$ - Equação 24 - é o resultado do balanço de dois tipos de energia: uma de volume, liberada para a formação do núcleo sólido $\left(\Delta \mathrm{G}_{\mathrm{v}}\right)$, e uma de superfície, gasta para a criação de uma interface sólido-líquido $\left(\Delta \mathrm{G}_{\mathrm{s}}\right)$.

$\Delta \mathrm{G}=\Delta \mathrm{G}_{\mathrm{v}}+\Delta \mathrm{G}_{\mathrm{s}}$

Para uma partícula de raio "r", a Equação 24 torna-se a Equação 25:

$\Delta \mathrm{G}=-\frac{4 \pi r^{3}}{3} \cdot \Delta \mathrm{G}_{\mathrm{v}}+4 \pi \mathrm{r}^{2} \cdot \varphi$

Em que:

$\varphi=$ tensão interfacial ou energia superficial $\left(\mathrm{J} \cdot \mathrm{m}^{-2}\right)$.

Valores de $\varphi$ variando de 15 a $50 \mathrm{~mJ} . \mathrm{m}^{-2}$ para a estruvita (KOFINA; KOUTSOUKOS, 2005; MPOUNTAS; PAPADAKIS; KOUTSOUKOS, 2017; BOUROPOULOS; KOUTSOUKOS, 2000; BHUYIAN; MAVINIC; BECKIE, 2008) têm sido encontrados na literatura.

Existe um ponto em que $\Delta \mathrm{G}$ tem o seu valor máximo positivo $\left(\Delta \mathrm{G}_{\text {crit }}\right)$, correspondente à formação do núcleo crítico. Portanto, a derivada da Equação 25, neste ponto, é igual a zero (Equação 26):

$\frac{\mathrm{d} \Delta \mathrm{G}}{\mathrm{dr}}=-4 \pi \mathrm{r}^{2} \Delta \mathrm{G}_{\mathrm{v}}+8 \pi \mathrm{r} \varphi=0$

E o raio crítico $\left(\mathrm{r}_{\text {crit }}\right)$ pode ser calculado pela Equação 27:

$\mathrm{r}_{\text {crit }}=\frac{2 \varphi}{\Delta \mathrm{G}_{\mathrm{v}}}$

A taxa de nucleação homogênea (J), isto é, o número de núcleos formados por unidade de tempo e de volume, pode ser expressa pela Equação 28

$\mathrm{J}=\Omega \cdot \exp \left(-\frac{\Delta \mathrm{G}}{\mathrm{K}_{\mathrm{B}} \cdot \mathrm{T}}\right)$

Em que:

$\mathrm{K}_{\mathrm{B}}=$ constante de Boltzmann $=1,3805 \cdot 10^{-23} \mathrm{~J} \cdot \mathrm{K}^{-1}$

Para um não eletrólito, é válida a equação de Gibbs-Thompson (Equação 29):

$\ln S=\frac{2 \cdot \varphi \cdot v}{\mathrm{~K}_{\mathrm{B}} \cdot \mathrm{T} \cdot \mathrm{r}}$

Em que:

$\mathrm{v}=$ volume molecular $\left(\mathrm{m}^{3}\right)=7,99 \cdot 10^{-23} \mathrm{~cm}^{3}$ para a estruvita;

Das Equações 27 a 29, obtém-se a Equação 30:

$\Delta \mathrm{G}_{\mathrm{v}}=\frac{2 \cdot \varphi}{r_{c}}=\frac{\mathrm{K}_{\mathrm{B}} \cdot \mathrm{T} \cdot \ln \mathrm{S}}{\mathrm{v}}$

Substituindo por $r=\operatorname{rcrite} \Delta G_{V}$ na Equação 25, obtém-se J (Equação 31):

$J=\Omega \cdot \exp \left[\frac{16 \pi \cdot \varphi^{3} \cdot v^{2}}{3 K_{B}^{3} \cdot T^{3} \cdot(\ln S)^{2}}\right]$ 
A Equação 31 pode ser generalizada para outros formatos de núcleos e para soluções eletrolíticas (Equação 32):

$J=\Omega \cdot \exp \left[\frac{-\beta \cdot \varphi^{3} \cdot v^{2}}{\mathrm{~K}_{\mathrm{B}}^{3} \cdot \mathrm{T}^{3} \cdot \mathrm{z}^{2} \cdot\left(\ln \mathrm{S}_{\mathrm{a}}\right)^{2}}\right]$

Em que:

$\beta=\frac{4 \mathrm{k}_{\mathrm{a}}^{3}}{27 \mathrm{k}_{\mathrm{v}}^{2}}=$ fator geométrico;

$\mathrm{k}_{\mathrm{a}}=$ fator de forma superficial;

$\mathrm{k}_{\mathrm{v}}$ = fator de forma volumétrico.

Segundo Nielsen (1964), $\Omega$ pode ser calculado pela Equação 33:

$\Omega=\left[\left(\frac{\mathrm{D}}{\mathrm{d}^{5} \cdot \mathrm{N}^{*}}\right) \cdot\left(\frac{4 \Delta \mathrm{G}^{*}}{3 \pi \mathrm{k}_{\mathrm{B}} \mathrm{T}}\right)^{\frac{1}{2}}\right]$

Na qual:

$\mathrm{D}=$ coeficiente de difusão $\left(\mathrm{m}^{2} \cdot \mathrm{s}^{-1}\right)$;

$\mathrm{D}=$ distância interplanar na estrutura do cristal (m);

$\mathrm{N}^{*}=$ número de moléculas ou pares de íons que formam o núcleo crítico (adimensional);

$\Delta \mathrm{G}^{*}=$ variação da energia livre de Gibbs para formar o núcleo crítico (J).

Nota-se pela Equação 32 que a taxa de nucleação homogênea é influenciada pela temperatura, pela razão de supersaturação e pela tensão interfacial. Um rápido aumento na taxa de nucleação ocorre quando o nível de supersaturação crítico é alcançado. Forrest et al. (2008) têm recomendado trabalhar com $\mathrm{S}_{\mathrm{a}}$ entre 1 e 2,5 e relação $\mathrm{Mg}: \mathrm{P}$ de 1 a 2,5.

O tempo que transcorre desde que se atinge a supersaturação até que se observe o núcleo crítico é conhecido como tempo de indução $\left(t_{\text {ind }}\right)$. Supondo que ele seja muito menor do que o tempo para o crescimento do núcleo, usando o conceito estatístico de nucleação, tem-se a Equação 34:

$\mathrm{t}_{\text {ind }}=\frac{1}{\mathrm{~J}}=\frac{1}{\Omega} \exp \left[\frac{\beta \cdot \mathrm{v}^{2} \cdot \varphi^{3}}{\mathrm{~K}_{\mathrm{B}}^{3} \cdot \mathrm{T}^{3} \cdot \mathrm{z}^{2} \cdot\left(\ln \mathrm{S}_{\mathrm{a}}\right)^{2}}\right]=\frac{1}{\Omega} \exp \left[\frac{\beta \cdot \mathrm{v}^{2} \cdot \varphi^{3}}{\left(2,3 \cdot \mathrm{K}_{\mathrm{B}} \mathrm{T}\right)^{3} \cdot \mathrm{z}^{2} \cdot\left(\log \mathrm{S}_{\mathrm{a}}\right)^{2}}\right]$

Portanto, o tempo de indução é altamente dependente da razão de supersaturação.

Na prática, dificilmente a nucleação homogênea ocorre, pois as águas residuárias têm impurezas.

\section{Nucleação primária heterogênea}

A nucleação primária heterogênea caracteriza-se por ser iniciada por partículas sólidas em suspensão diferentes da estruvita (impurezas). Nesse caso, os clusters adsorvem na(s) superfície(s) da(s) impureza(s). A área da interface impureza-cluster substitui uma quantidade equivalente de área da interface impureza-líquido. A tensão interfacial impureza-cluster é menor do que a tensão impureza-líquido, desde que a superfície da impureza seja molhada pela nova fase nucleada. Portanto, o aparecimento da superfície impureza-cluster reduz a energia associada com a área que a impureza ocupa no líquido. Dessa forma, a barreira energética a ser vencida na nucleação heterogênea é muito menor do que aquela na nucleação homogênea e, portanto, ela ocorre em um grau de supersaturação bem menor (MULLIN, 2001). Consequentemente, o tempo de indução, expresso pela Equação 34, é reduzido e pode ser calculado pela Equação 35:

$\operatorname{logt}_{\text {ind }}=\left[\frac{\mathrm{A}}{\left(\ln \mathrm{S}_{\mathrm{a}}\right)^{2}}\right]-B$

Em que:

$A=\frac{\beta \cdot v^{2} \cdot \varphi^{3} \cdot f(\theta)}{\left(2,3 \cdot K_{B} T\right)^{3} \cdot z^{2}}$

$B=\log \Omega$ se $f(\theta)=1$ e $\log \Omega_{\text {het }}$ se $f(\theta)<1$;

$f(\theta)=\frac{\left(2-3 \cos \theta+\cos ^{3} \theta\right)}{4}=$ fator de correção para a nucleação heterogênea;

$\Theta=$ ângulo de molhamento;

$\Omega_{\text {het }}=$ fator pré-exponencial de nucleação heterogênea.

Mehta e Batstone (2013) pesquisaram a nucleação primária em função da razão de supersaturação em água sintética e em sobrenadante de digestor anaeróbio de sistema EBPR. Eles concluíram que quando a $S_{a}$ foi menor do que 1,7 , ocorreu a nucleação heterogênea, a partir de impurezas microscópicas de tamanho menor que $0,45 \mu \mathrm{m}$. Para $S_{a}>1,7$, a nucleação foi homogênea (Figura 2). Bouropoulos e Koutsoukos (2000) obtiveram S de 2,0 e Hanhoun et al. (2013), de 2,68.

Na Figura 2, verifica-se grande variação nos resultados obtidos por diferentes pesquisadores, consequência da forma de medição usada para determinar $\mathrm{t}_{\text {ind }}$ (alteração de $\mathrm{pH}$, de turbidez, de condutividade, de absorbância, de intensidade de luz etc.). Assim, o tempo de indução não pode ser usado como parâmetro de projeto.

Observa-se, também, que a nucleação homogênea ocorre em alta razão de supersaturação, devido a uma maior interação molecular.

Ohlinger, Young e Schroeder (1999), Bhuyian, Mavinic e Beckie (2008) e Mehta e Batstone (2013) concluíram que a intensidade de mistura não tinha uma influência no $t_{\text {ind }}$ tão significativa quanto a $S_{a}$ na nucleação heterogênea.

Uma implicação prática dos conceitos aqui apresentados é que em um cristalizador há zonas com alta razão de supersaturação, como, por exemplo, na alimentação, em que as concentrações de magnésio e de álcali são maiores, ou em locais nos quais a turbulência causa volatilização do $\mathrm{CO}_{2}$, próximos a bombas e agitadores, que elevam o $\mathrm{pH}$ e 
reduzem a solubilidade da estruvita. Nesses locais, o tempo de indução é baixo e formam-se muitos cristais pequenos, que são difíceis de serem separados e que causam incrustação. Para resolver o problema, alguns pesquisadores têm recomendado o uso de coagulantes/floculantes (LE CORRE et al., 2007), a filtração (ETTER et al., 2011), a limitação na velocidade ascensional de cristalizadores de leito fluidificado, a maior relação diâmetro/altura do reator (FATTAH; MAVINIC; KOCH, 2012) e a recirculação dos finos (UENO; FUJII, 2001; SHIMAMURA et al., 2003; FORREST et al., 2008).

\section{Nucleação secundária}

A nucleação secundária só ocorre quando cristais já estão presentes no meio. Nesse tipo de nucleação, o contato pode ser entre os cristais existentes, entre os cristais e as paredes e outras partes internas do reator ou entre os cristais e o agitador mecânico.

Denomina-se semeadura quando se adicionam cristais ao sistema de forma intencional ou acidental. Ela deve ser introduzida na zona metaestável (região entre as curvas de solubilidade e de supersaturação), em que ocorre o fenômeno denominado "coalescimento de Ostwald", no qual os íons que estão na vizinhança dos núcleos menores são deslocados para a superfície dos maiores, por diferença de concentrações, dissolvendo os menores e aumentando os maiores. Embora Galbraith e Schneider (2009) tenham mostrado que, mesmo em razões de supersaturação baixas $\left(1,0<\mathrm{S}_{a}<1,35\right)$, ainda ocorra a nucleação homogênea, ela não é significativa. Portanto, essa é uma estratégia para resolver o problema citado dos finos.

Os mecanismos pelos quais ocorre a nucleação secundária ainda não foram completamente entendidos. Segundo recente revisão conduzida por Agrawal e Paterson (2015), a nucleação pode ocorrer por colisão ou por cisalhamento do fluido, que produz atrito ou remoção da camada superficial da semente. As colisões ocorrem entre o núcleo e a semente ou entre o núcleo e o agitador, sendo controladas, respectivamente, na microescala (microvórtices) e na mesoescala (hidrodinâmica).

A taxa de nucleação secundária (Ns), em número de núcleos produzidos por volume de solução na unidade de tempo, pode ser calculada pela Equação 36:

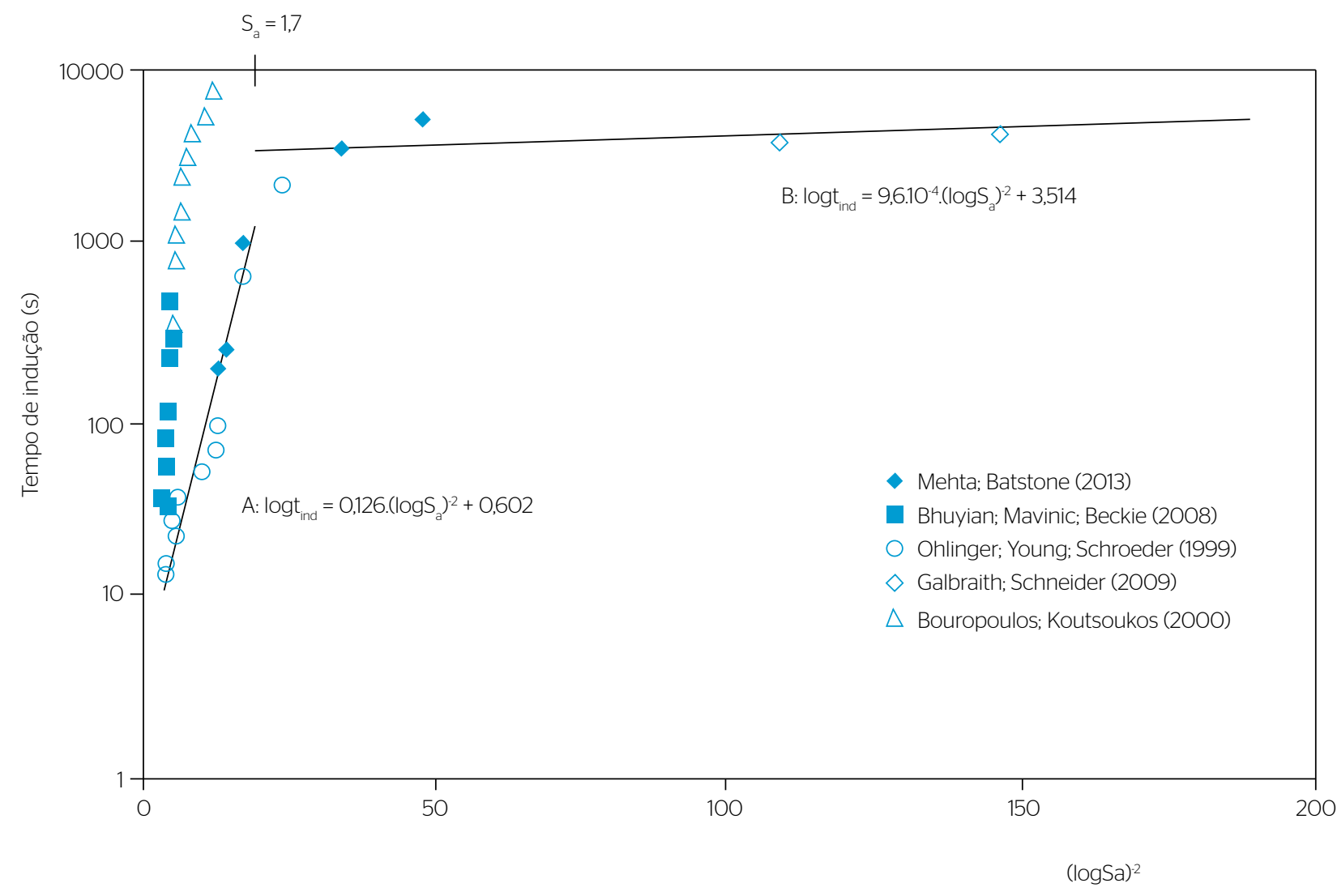

Fonte: adaptado de Mehta e Batstone (2013)

Figura 2 - Tempo de indução em função da razão de supersaturação obtidos por diferentes autores. 
$\mathrm{N}_{\mathrm{S}}=\mathrm{K}_{\mathrm{S}} \cdot \mathrm{n}^{\mathrm{j}} \cdot \mathrm{M}_{\mathrm{T}}^{\mathrm{k}} \cdot \sigma^{\mathrm{n}_{\mathrm{s}}}$

Em que:

$\mathrm{K}_{\mathrm{S}}=$ constante de taxa de nucleação secundária (núcleos. $\mathrm{L}^{-1} \cdot \mathrm{S}^{-1}$ );

$\mathrm{N}=$ rotação do agitador (rps);

$\mathrm{M}_{\mathrm{T}}=$ densidade de sementes na suspensão ( $\mathrm{kg}$ de sementes. $\mathrm{L}^{-1}$ de suspensão);

$\sigma$ : supersaturação relativa; e

$\mathrm{j}$, k e $\mathrm{n}_{\mathrm{s}}=$ constantes empíricas.

Poucos são os pesquisadores que se dedicaram a estudar a nucleação secundária na cristalização da estruvita. Eles simplificaram a Equação 36 para a Equação 37:

$\mathrm{N}_{\mathrm{S}}^{*}=\mathrm{K}_{\mathrm{S}}^{*} \cdot \sigma^{\mathrm{n}_{\mathrm{S}}^{*}}$

Os valores de $\mathrm{n}_{\mathrm{S}}^{*}$ e $\mathrm{K}_{\mathrm{S}}^{*}$ obtidos por Mehta e Batstone (2013) e Galbraith e Schneider (2014) foram, respectivamente, 1,75 \pm 0,13 e $8,3 \pm 2,3 \cdot 10^{6}$ núcleos. $\mathrm{L}^{-1} \cdot \mathrm{s}^{-1}$ e $1,680 \pm 0,014$ e $1,4 \cdot 10^{6}$ núcleos. $\mathrm{L}^{-1} \mathrm{~s}^{-1}$, e estão bastante próximos.

A Equação 36 mostra que o nível de supersaturação, a intensidade de mistura e a quantidade de semente têm influência significativa na nucleação secundária (BHUYIAN; MAVINIC; BECKIE, 2008; MEHTA; BATSTONE, 2013; GALBRAITH; SCHNEIDER, 2014; MPOUNTAS; PAPADAKIS; KOUTSOUKOS, 2017). Mehta e Batstone (2013) verificaram que em supersaturação relativa abaixo de $0,55 \pm 0,1$ não ocorria a nucleação. Em uma mesma razão de supersaturação, Mpountas, Papadakis e Koutsoukos (2017) observaram que aumentando a concentração de sementes de estruvita, aumentava-se a taxa de nucleação. Eles atribuíram tal comportamento à quebra das sementes pela agitação.

Diferentes materiais têm sido utilizados para a semeadura, tais como finos da estruvita, vidro borossilicato, areia (REGY et al., 2002; ALI, 2005; LE CORRE et al., 2007; ALI; SCHNEIDER, 2005), sendo o primeiro o mais empregado. Quando são adicionados finos da estruvita na solução, não há barreira energética a ser rompida nem tempo de indução, o que evita a nucleação primária e a formação de fases instáveis termodinamicamente. Como a área superficial aumenta com a introdução dos finos, o processo de adsorção dos íons formadores da estruvita é favorecido, $\mathrm{o}$ que causa melhoria na recuperação do fósforo (LIU et al., 2008; ZHANG et al., 2009; LIU et al., 2011; YU et al., 2013). Quando se usa materiais com estruturas cristalinas muito diferentes da estruvita, os novos núcleos não conseguem se integrar às sementes para o crescimento do cristal, resultando em embriões (RAHAMAN; ELLIS; MAVINIC, 2008; WANG; BURKEN; ZHANG, 2006; REGY et al., 2002; ALI, 2005; BATTISTONI et al., 2000) e aumentando o tempo de indução.

\section{CONSIDERAÇÕES FINAIS}

O setor de saneamento básico brasileiro deve ampliar sua visão: é necessário pensar no esgoto como fonte de matérias-primas, e não apenas como descarte, de acordo com os modernos preceitos da ecologia industrial e da economia circular. Assim, em vez da simples remoção de nutrientes, deve-se incentivar a sua recuperação, seja pela possibilidade de exaustão das reservas naturais (caso do fósforo e do potássio), seja pela baixa eficiência no seu aproveitamento e pelos impactos ambientais (caso do nitrogênio). Nesse sentido, deve-se evitar lançar no sistema público de esgoto tudo que impeça essa recuperação, como, por exemplo, águas residuárias industriais, lodo de estação de tratamento de água, lixiviado de aterro etc.

Os benefícios do uso da estruvita na agricultura são conhecidos desde a década de 1960, entretanto esse tema voltou a ganhar importância apenas nos últimos anos, por conta de algumas previsões indicarem que a duração das rochas fosfatadas do planeta seja de, no máximo, 400 anos. Apesar de a cristalização ser um tema bastante estudado em outras áreas de conhecimento, sua utilização no saneamento básico é recente. Consequentemente, a recuperação de nutrientes do esgoto como cristais de estruvita ainda não é uma tecnologia totalmente dominada. Como tópicos que necessitam ser pesquisados, podem-se citar:

- produção de estruvita por meio biológico ou biológico/químico;

- desenvolvimento de modelos de previsão robustos, que incorporem a termodinâmica, a cinética, a transferência de massa e a hidrodinâmica;

- validação dos modelos em escala real;

- melhor entendimento da nucleação, especialmente a secundária, que ocorre na maioria dos cristalizadores em escala real;

- estratégias para melhoria da qualidade da estruvita produzida.

\section{AGRADECIMENTOS}

Ao Conselho Nacional de Desenvolvimento Científico e Tecnológico $(\mathrm{CNPq})$, pela concessão de bolsa de mestrado; e à Fundação de Amparo à Pesquisa do Estado de São Paulo (FAPESP), pelo financiamento do projeto.

\section{FONTE DE FINANCIAMENTO}

Conselho Nacional de Desenvolvimento Científico e Tecnológico (CNPq) e Fundação de Amparo à Pesquisa do Estado de São Paulo (FAPESP). 


\section{REFERÊNCIAS}

AAGE, H.K.; ANDERSEN, B.L.; BLOM, A.; JENSEN, I. (1997) The solubility of struvite. Journal of Radioanalytical and Nuclear Chemistry, v. 223, n. 1-2, p. 213-215. https://doi.org/10.1007/BFO2223387

ABBONA, F.; BOISTELLE, R. (1979) Growth morphology and crystal habit of struvite crystals (MgNH4PO4 $6 \mathrm{H} 2 \mathrm{O}$ ). Journal of Crystal Growth, v. 46, n. 3, p. 339-354. https://doi.org/10.1016/0022O248(79)90082-4

ADNAN, A.; MAVINIC, D.S.; KOCH, F.A. (2003) Pilot-scale study of phosphorus recovery through struvite crystallization - examining the process feasibility. Journal of Environmental Engineering and Science, v. 2, n. 5, p. 315-324. https://doi.org/10.1139/s03-040

AGRAWAL, S.G.; PATERSON, A.H.J. (2O15) Secondary nucleation: mechanisms and models. Chemical Engineering Communications, v. 202, n. 5, p. 698-706. https://doi.org/101080/00986445.2014.969369

ALEXANDRATOS, N.; BRUINSMA, J. (2012) World Agriculture Towards 2030/2050: The 2012 Revision. ESA Working Paper, n. 12-03. Agricultural Development Economics Division. Roma: FAO. Disponível em: <http://www.fao.org/docrep/016/ap106e/ap106e.pdf>. Acesso em: 16 jan. 2013.

ALI, M.I. (2005) Struvite crystallization from nutrient rich wastewater. 271f. PhD Thesis (Doctoring) - School of Engineering, James Cook University, Austrália. Disponível em: <https://researchonline.jcu.edu. au/148/2/O2whole.pdf>. Acesso em: 25 jun. 2012.

(2007) Struvite crystallization in fed-batch pilot scale and description of solution chemistry of struvite. Chemical Engineering Research and Design, v. 85, n. 3, p. 344-356. http://dx.doi.org/10.1205/ cherd06031

ALI, M.I.; SCHNEIDER, P.A. (2005) Crystallization of struvite from metastable region with different types of seed crystal. Journal of Non-Equilibrium Thermodynamics, v. 30, n. 2, p. 95-111. https://doi. org/10.1515/JNETDY.2005.007

ALI, M.I.; SCHNEIDER, P.A.; HUDSON, N. (2003) Nutrient recovery from piggery effluents. Engineering Modelling, v. 16, n. 1-2, p. 71-76.

ANDRADE, A.; SCHUILING, R.D. (2001) The chemistry of struvite crystallization. Mineralogical Journal, v. 23, n. 5-6, p. 37-46.

BABIC-IVANCIC, V.; KONTREC, J.; KRALJ, D.; BRECEVIC, L. (2002) Precipitation diagrams of struvite and dissolution kinetics of different struvite morphologies. Croatica Chemica Acta, v. 75, n. 1, p. 89-106.

BATTISTONI, P.; BOCCADORO, R.; FATONE, F.; PAVAN, P. (2005) Autonucleation and crystal growth of struvite in a demonstrative fluidized bed reactor (FBR). Environmental Technology, v. 26, n. 9, p. 975-982. https://doi.org/10.1080/09593332608618486

BATTISTONI, P.; PAVAN, P.; PRISCIANDARO, M.; CECCHI, F. (2OOO) Struvite crystallization: A feasible and reliable way to fix phosphorus in anaerobic supernatants. Water Research, v. 34, n. 11, p. 3033-3041. http://dx.doi.org/10.1016/S0043-1354(00)00045-2

BHUYIAN, M.I.H.; MAVINIC, D.S.; BECKIE, R.D. (2007) A Solubility and thermodynamic study of struvite. Environmental Technology, v. 28, n. 9. p. 1015-1026. https://doi.org/10.1080/09593332808618857
BHUYIAN, M.I.H.; MAVINIC, D.S.; BECKIE, R.D. (2008) Nucleation and growth kinetics of struvite in a fluidized bed reactor. Journal of Crystal Growth, v. 310, n. 6, p. 1187-1194. http://dx.doi.org/10.1016/j. jcrysgro.2007.12.054

BHUYIAN, M.I.H.; MAVINIC, D.S.; BECKIE, R.D. (2009) Determination of temperature dependence of electrical conductivity and its relationship with ionic strength of anaerobic digester supernatant, for Struvite Formation. Journal of Environmental Engineering, v. 135 , n. 11 , p. 1221-1226. https://doi.org/10.1061/(ASCE)07339372(2009)135:11(1221)

BITTON, G. (2005) Wastewater microbiology. Hoboken: John Wiley $\&$ Sons.

BOUROPOULOS, N.C: KOUTSOUKOS, PG. (2000) Spontaneous precipitation of struvite from aqueous solutions. Journal of Crysta Growth, v. 213, n. 3-4, p. 381-388

BRIDGER, G.L.; SALUTSKY, M.L.;STAROSTKA, R.W. (1962) Micronutrient sources, metal ammonium phosphates as fertilizers. Journal of Agricultural and Food Chemistry, v. 10, n. 3, p. 181-188. https://doi. org/10.1021/jf60121aO06

BRITTON, A.; KOCH, F.A.; MAVINIC, D.S.; ADNAN, A.; OLDHAM, W.K. UDALA, B. (2005) Pilot-scale struvite recovery from anaerobic digester supernatant at an enhanced biological phosphorus removal wastewater treatment plant. Journal of Environmental Engineering and Science, v. 4, n. 4, p. 265-277. https://doi.org/10.1139/s04-059

BUCHANAN, J.; MOTE, C.; ROBINSON, R. (1994) Thermodynamics of struvite formation. Transactions of the ASAE, v. 37, n. 2, p. 617-621 https://doi.org/10.13031/2013.28121

BURNS, J.R.; FINLAYSON, B. (1982) Solubility product of magnesium ammonium phosphate hexahydrate at various temperatures. The Journal of Urology, v. 128, n. 2, p. 426-428. https://doi.org/10.1016/ SOO22-5347(17)52952-3

CALVO, G.; VALERO, A.; VALERO, A. (2017) Assessing maximum production peak and resource availability of non-fuel mineral resources: Analyzing the influence of extractable global resources. Resources, Conservation and Recycling, v. 125, p. 208-217. http:// dx.doi.org/10.1016/j.resconrec.2017.06.009

ÇELEN, I.; BUCHANAN, J.R.; BURNS, R.T.; ROBINSON, R.B.; RAMAN, D.R. (2007) Using a chemical equilibrium model to predict amendments required to precipitate phosphorus as struvite in liquid swine manure. Water Research, v. 41, n. 8, p. 1689-1696. https://doi.org/10.1016/j. watres.2007.01.018

CERRILLO, M.; PALATSI, J.; COMAS, J.; VICENS, J.; BONMATÍ, A. (2015) Struvite precipitation as a technology to be integrated in a manure anaerobic digestion treatment plant - removal efficiency, crystal characterization and agricultural assessment. Journal of Chemical Technology and Biotechnology, v. 90, n. 6, p. 1135-1143. https://doi org/10.1002/jctb.4459

CHENG, H.; XU, W.; LIU, J.; ZHAO, Q.; HE, Y:; CHEN, G. (2007) Application of composted sewage sludge (CSS) as a soil amendment for turfgrass growth. Ecological Engineering, v. 29, n. 1, p. 96-104. http:// dx.doi.org/10.1016/j.ecoleng.2006.08.005 
CHERKASOV, N.; IBHADON, A.O.; FITZPATRICK, P. (2O15) A review of the existing and alternative methods for greener nitrogen fixation. Chemical Engineering and Processing: Process Intensification, v. 90, p. 24-33. https://doi.org/10.1016/j.cep.2015.02.004

CORDELL, D.; WHITE, S. (2014) Life's Bottleneck: Sustaining the world's phosphorus for a food secure future. Annual Review of Environment and Resources, v. 39, p. 161-188. https://doi.org/10.1146/ annurev-environ-010213-113300

CORNEL, P.; SCHAUM, C. (2009) Phosphorus recovery from wastewater: needs, technologies and costs. Water Science and Technology, v. 59, n. 6, p. 1069-1076. https://doi.org/10.2166/ wst.2009.045

CRUTCHIK, D.; GARRIDO, J.M. (2011) Struvite crystallization versus amorphous magnesium and calcium phosphate precipitation during the treatment of a saline industrial wastewater. Water Science and Technology, v. 64, n. 12, p. 2460-2467. https://doi.org/10.2166/ wst.2011.836

CRUTCHIK, D.; GARRIDO, J.M. (2016) Kinetics of the reversible reaction of struvite crystallisation. Chemosphere, v. 154, p. 567-572. https://doi. org/10.1016/j.chemosphere.2016.03.134

CRUTCHIK, D.; SÁNCHEZ, A.; GARRIDO, J.M. (2013) Simulation and experimental validation of multiple phosphate precipitates in a saline industrial wastewater. Separation and Purification Technology, v. 118, n. 30, p. 81-88. https://doi.org/10.1016/j.seppur.2013.06.041

DESMIDT, E.; GHYSELBRECHT, K.; ZHANG, Y.; PINOY, L.; VAN DER BRUGGEN, B.; VERSTRAETE, W.; RABAEY, K.; MEESSCHAERT, B. (2O15) Global phosphorus scarcity and full-scale P-recovery techniques: a review. Critical Reviews in Environmental Science and Technology, v. 45, n. 4, p. 336-384. https://doi.org/10.1080/10643389.2013.866531

DOYLE, J.D.; PARSONS, S.A. (2002) Struvite formation, control and recovery. Water Research, v. 36, n. 16, p. 3925-3940.

DOYLE, J.D.; PHILIP, R.; CHURCHLEY, J.; PARSONS, S.A. (2000) Analysis of struvite precipitation in real and synthetic liquors. Process Safety and Environmental Protection, v. 78, n. 6, p. 480-488. https:// doi.org/10.1205/095758200531023

ERISMAN, J.W.; SUTTON, M.A.; GALLOWAY, J.; KLIMONT, Z; WINIWARTER, W. (2008) How a century of ammonia synthesis changed the world. Nature Geoscience, v. 1, n. 10, p. 636-639.

ETTER, B.; TILLEY, E.; KHADKA, R.; UDERT, K.M. (2011) Low-cost struvite production using source-separated urine in Nepal. Water Research, $v$. 45, n. 2, p. 852-862. https://doi.org/10.1016/j.watres.2010.10.007

FATTAH, K.P.; MAVINIC, D.S.; KOCH, F.A. (2O12) Influence of process parameters on the characteristics of struvite pellets. Journal of Environmental Engineering, v. 138, n. 12, p. 1200-1209. https://doi. org/10.1061/(ASCE)EE.1943-7870.0000576

FLORES-ALSINA, X.; SOLON, K.; MBAMBA, C.K.; TAIT, S.; GERNAEY, K.V.; JEPPSSON, U.; BATSTONE, D.J. (2016) Modelling phosphorus $(\mathrm{P})$, sulfur (S) and iron (Fe) interactions for dynamic simulations of anaerobic digestion processes. Water Research, v. 95, n. 1, p. 370-382. https://doi.org/10.1016/j.watres.2016.03.012

FORREST, A.L.; FATTAH, K.P.; MAVINIC, D.S.; KOCH, F.A. (2OO8) Optimizing struvite production for phosphate recovery in WWTP. Journal of Environmental Engineering, v. 134, n. 5, p. 395-402. https:// doi.org/10.1061/(ASCE)0733-9372(2008)134:5(395)
GALBRAITH, S.C.; SCHNEIDER, P.A. (2009) A review of struvite nucleation studies. In: INTERNATIONAL CONFERENCE ON NUTRIENT RECOVERY FROM WASTEWATER STREAMS. Anais.. Vancouver: IWA. n. 1, p. 69-78.

GALBRAITH, S.C.; SCHNEIDER, P.A. (2014) Modelling and simulation of inorganic precipitation with nucleation, crystal growth and aggregation: A new approach to an old method. Chemical Engineering Journal, v. 240, p. 124-132. http://dx.doi.org/10.1016/j. cej.2013.11.070

GALLOWAY, J.N.; ABER, J.D.; ERISMAN, J.W.; SEITZINGER, S.P.; HOWARTH, R.W.; COWLING, E.B.; COSBY, B.J. (2003) The Nitrogen Cascade. BioScience, v. 53, n. 4, p. 341-356. https://doi.org/10.1641/00063568(2003)053[0341:TNC]2.0.CO;2

GUEDES, M.C. (2005) Ciclagem de nutrientes após aplicação de lodo de esgoto (biossólido) sobre latossolo cultivado com Eucalyptus Grandis. 154f. Tese (Doutorado) - Escola Superior de Agricultura Luiz de Queiroz, Universidade de São Paulo, São Paulo. Disponível em: <http://www.teses.usp.br/teses/disponiveis/86/86131/tde-19012012161545/pt-br.php>. Acesso em: 5 jan. 2014.

HANHOUN, M.; MONTASTRUC, L.; AZZARO-PANTEL, C.; BISCANS, B.; FRECHE, M.; PIBOULEAU, L. (2O11) Temperature impact assessment on struvite solubility product : a thermodynamic modeling approach. Chemical Engineering Journal, v. 167, n. 1, p. 50-58. http://dx.doi. org/10.1016/j.cej.2010.12.001

HANHOUN, M.; MONTASTRUC, L.; AZZARO-PANTEL, C.; BISCANS, B.; FRECHE, M.; PIBOULEAU, L. (2013) Simultaneous determination of nucleation and crystal growth kinetics of struvite using a thermodynamic modeling approach. Chemical Engineering Journal, v. 215-216, p. 903-912. https://doi.org/10.1016/j. cej.2012.10.038

HAO, X.D.; WANG, C.; LAN, L.; VAN LOOSDRECHT, M.C.M. (2008) Struvite formation, analytical methods and effects of $\mathrm{pH}$ and $\mathrm{Ca} 2+$ Water Science and Technology, v. 58, n. 8, p. 1687-1692. https://doi. org/10.2166/wst.2008.557

HAO, X.D.; WANG, C.; VAN LOOSDRECHT, M.C.M.; HU, Y. (2O13) Looking beyond struvite for P-recovery. Environmental Science and Technology, v. 47, n. 10, p. 4965-4966. https://doi.org/10.1021/es401140s

HECKENMÜLLER, M.; NARITA, D.; KLEPPER, G. (2014) Global availability of phosphorus and its implications for global food supply: an economic overview. Kiel Working Paper, n. 1897. Disponível em: <https://www.ifw-members.ifw-kiel.de/publications/ global-availability-of-phosphorus-and-its-implications-for-globalfood-supply-an-economic-overview/KWP 1897.pdf>. Acesso em: 28 nov. 2015

HUANG, H.; XIAO, D.; LIU, J.; HOU, L.; DING, L. (2015) Recovery and removal of nutrients from swine wastewater by using a novel integrated reactor for struvite decomposition and recycling. Scientific Reports, v. 5, p. 1-13. https://dx.doi.org/10.1038\%2Fsrep10183

HUCHZERMEIER, M.P.; TAO, W. (2O12) Overcoming Challenges to Struvite Recovery from Anaerobically Digested Dairy Manure. Water Environment Research, v. 84, n. 1, p. 34-41.

HUTNIK, N.; WIERZBOWSKA, B.; PIOTROWSKI, K.; MATYNIA, A (2013) Effect of Copper (II) Ions on Quality of Struvite Produced in Continuous Reaction Crystallization Process at the Magnesium lons Excess. Advances in Chemical Engineering and Science, v. 3, n. 4B, p. 1-6. http://dx.doi.org/10.4236/aces.2013.34BO01 
HUTNIK, N.; WIERZBOWSKA, B.; PIOTROWSKI, K.; MATYNIA, A. (2O16) Effect of continuous crystallizer performance on struvite crystals produced in reaction crystallization from solutions containing phosphate(V) and ZINC(II) IONS. Brazilian Journal of Chemical Engineering, v. 33, n. 2, p. 307-317. http://dx.doi.org/101590/0104$6632.20160332 \mathrm{~s} 00003385$

INTERGOVERNMENTAL TECHNICAL PANEL OF SOILS (ITPS). (2015) Status of the world's soil resources. Roma: FAO. Disponível em: <http:// www.fao.org/3/a-i5199e.pdf>. Acesso em: 10 fev. 2016.

KABDASZLI, I.; PARSONS, S.A.; TÜNAYA, O. (2006) Effect of major ions on induction time of struvite precipitation. Croatica Chemica Acta, v. 79, n. 2, p. 243-251.

KATAKI, S.; WEST, H.; CLARKE, M.; BARUAH, D.C. (2016a) Phosphorus recovery as struvite: Recent concerns for use of seed, alternative $\mathrm{Mg}$ source, nitrogen conservation and fertilizer potential. Resources, Conservation and Recycling, v. 107, p. 142-156. https://doi.org/10.1016/j. resconrec.2015.12.009

KATAKI, S.; WEST, H.; CLARKE, M.; BARUAH, D.C. (2016b) Phosphorus recovery as struvite from farm, municipal and industrial waste: Feedstock suitability, methods and pre-treatments. Waste Management, v. 49, p. 437-454. https://doi.org/10.1016/j. wasman.2016.01.003

KOFINA, A.N.; KOUTSOUKOS, P.G. (2005) Spontaneous precipitation of struvite from synthetic wastewater solutions. Crystal Growth \& Design, v. 5, n. 2, p. 489-496. https://doi.org/10.1021/cgO49803e

KOZIK, A.; HUTNIK, N.; MATYNIA, A.; GLUZINSKA, J.; PIOTROWSKI, K. (2011) Recovery of phosphate $(V)$ ions from liquid waste solutions containing organic impurities. CHEMIK, v. 65, n. 7, p. 675-686.

KOZIK, A.; HUTNIK, N.; PIOTROWSKI, K.; MAZIENCZUK, A.; MATYNIA, A. (2013) Precipitation and crystallization of struvite from synthetic wastewater under stoichiometric conditions. Advances in Chemical Engineering and Science, v. 3, n. 4, p. 20-26. http://dx.doi.org/10.4236/ aces.2013.34B004

LE CORRE, K.S.; VALSAMI-JONES, E.; HOBBS, P.; JEFFERSON, B.; PARSONS, S.A. (2007) Agglomeration of struvite crystals. Water Research, v. 41, n. 2, p. 419-425. https://doi.org/10.1016/j. watres.2006.10.025

LE CORRE, K.S.; VALSAMI-JONES, E.; HOBBS, P.; PARSONS, S.A. (2005) Impact of calcium on struvite crystal size, shape and purity. Journal of Crystal Growth, v. 283, n. 3-4, p. 514-522. https://doi.org/10.1016/j. jcrysgro.2005.06.012

LE CORRE, K.S.; VALSAMI-JONES, E.; HOBBS, P.; PARSONS, S.A. (2009) Phosphorus recovery from wastewater by struvite crystallization: a review. Critical Reviews in Environmental Science and Technology, v. 39, n. 6, p. 433-477. https://doi. org/10.1080/10643380701640573

LEE, S.H.; KUMAR, R.; JEON, B.-H. (2016) Struvite precipitation under changing ionic conditions in synthetic wastewater: Experiment and modeling. Journal of Colloid and Interface Science, v. 474, p. 93-102. https://doi.org/10.1016/j.jcis.2016.04.013

LEE, S.H.; YOO, B.-H.; LIM, S.J.; KIM, T.-H.; KIM, S.-K.; KIM, J.Y. (2O13) Development and validation of an equilibrium model for struvite formation with calcium co-precipitation. Journal of Crystal Growth, v. 372, p. 129-137. https://doi.org/10.1016/j.jcrysgro.2013.03.010
LIN, Y.M.; BASSIN, J.P.; VAN LOOSDRECHT, M.C.M. (2012) The contribution of exopolysaccharides induced struvites accumulation to ammonium adsorption in aerobic granular sludge. Water Research, v. 46, n. 4, p. 986-992. https://doi.org/10.1016/j.watres.2011.11.072

LIU, Y.H.; KWAG, J.-H.; KIM, J.-H.; RA, C.S. (2011) Recovery of nitrogen and phosphorus by struvite crystallization from swine wastewater Desalination, v. 277, n. 1, p. 364-369. http://dx.doi.org/10.1016/j. desal.2011.04.056

LIU, Z.; ZHAO, Q.; LEE, D.J.; YANG, N. (2008) Enhancing phosphorus recovery by a new internal recycle seeding MAP reactor. Bioresource Technology, v. 99, n. 14, p. 6488-6493. http://dx.doi.org/10.1016/j. biortech.2007.11.039

MAURER, M.; SCHWEGLER, P.; LARSEN, T.A. (2003) Nutrients in urine: energetic aspects of removal and recovery. Water Science and Technology, v. 48, n. 1, p. 37-46. https://doi.org/10.2166/wst.2003.0011

MEHTA, C.M.; BATSTONE, D.J. (2013) Nucleation and growth kinetics of struvite crystallization. Water Research, v. 47, n. 8, p. 2890-2900. https://doi.org/10.1016/j.watres.2013.03.007

MELIA, P.M.; CUNDY, A.B.; SOHI, S.P.; HOODA, P.S.; BUSQUETS, R. (2017) Trends in the recovery of phosphorus in bioavailable forms from wastewater. Chemosphere, v. 186, p. 381-395. https://doi.org/10.1016/j. chemosphere.2017.07.089

MPOUNTAS, I.; PAPADAKIS, E.; KOUTSOUKOS, P. (2017) Phosphorus recovery from simulated municipal wastewater (SMW) through the crystallization of magnesium ammonium phosphate hexahydrate (MAP). Journal of Chemical Technology and Biotechnology, v. 92, n. 8, p. 2075-2082. https://doi.org/10.1002/jctb.5202

MULLIN, JW. (2001) Crystallization. 4. ed. Londres: Butterworth Heinemann.

MÜNCH, E.V.; BARR, K. (2001) Controlled struvite crystallisation for removing phosphorus from anaerobic digester sidestreams. Water Research, v. 35, n. 1, p. 151-159.

MUSTER, T.H.; DOUGLAS, G.B.; SHERMAN, N.; SEEBER, A.; WRIGHT, N.; GÜZÜKARA, Y. (2013) Towards effective phosphorus recycling from wastewater: Quantity and quality. Chemosphere, v. 91, n. 5, p. 676-684. https://doi.org/10.1016/j.chemosphere.2013.01.057

MUSVOTO, E.V.; EKAMA, G.A.; WENTZEL, M.C.; LOEWWENTHAL, R.E. (2000) Extension and application of the three-phase weak acid/base kinetic model to the aeration treatment of anaerobic digester liquors. Water SA, v. 26, n. 4, p. 417-438.

NELSON, N.O.; MIKKELSEN, R.L;; HESTERBERG, D.L. (2003) Struvite precipitation in anaerobic swine lagoon liquid: effect of $\mathrm{pH}$ and $\mathrm{Mg}: \mathrm{P}$ ratio and determination of rate constant. Bioresource Technology, $\mathrm{v}$. 89, n. 3, p. 229-236

NIELSEN, A.E. (1964) Kinetics of precipitation. Oxford: Pergamon.

OHLINGER, K.N.; YOUNG, T.M.; SCHROEDER, E.D. (1998) Predicting struvite formation in digestion. Water Research, v. 32, n. 12, p. 36073614. http://dx.doi.org/10.1016/S0043-1354(98)00123-7

OHLINGER, K.N.; YOUNG, T.M.; SCHROEDER, E.D. (1999) Kinetics effects on preferential struvite accumulation in wastewater. Journal of Environmental Engineering, v. 125, n. 8, p. 730-737. https://doi org/10.1061/(ASCE)0733-9372(1999)125:8(730) 
OUCHAH, L.; MANDI, L.; BERREKHIS, F.; OUAZZANI, N. (2014) Essays of phosphorus recovery into struvite from fertilizer industry effluents. Desalination and Water Treatment, v. 52, n. 13-15, p. 28862892. https://doi.org/10.1080/19443994.2013.807080

PARSONS, S.A; SMITH, J.A. (2008) Phosphorus Removal and Recovery from Municipal Wastewater. Elements, v. 4, n. 2, p. 109-112. http://dx.doi.org/10.2113/GSELEMENTS.4.2.109

RAHAMAN, M.S.; ELLIS, N.; MAVINIC, D.S. (2008) Effects of various process parameters on struvite precipitation kinetics and subsequent determination of rate constants. Water Science and Technology, v. 57, n. 5, p. 647-654. https://doi.org/10.2166/wst.2008.022

RAHAMAN, M.S.; MAVINIC, D.S.; BHUYIAN, M.I.H.; KOCH, F.A. (2OO6) Exploring the determination of struvite solubility product from analytical results. Environmental Technology, v. 27, n. 9, p. 951-961. https://doi.org/10.1080/09593332708618707

RAHAMAN, M.S.; MAVINIC, D.S.; MEIKLEHAM, A.; ELLIS, N. (2014) Modeling phosphorus removal and recovery from anaerobic digester supernatant through struvite crystallization in a fluidized bed reactor. Water Research, v. 51, p. 1-10. https://doi.org/10.1016/j.watres.2013.11.048

RAWN, A.M.; BANTA, A.P.; POMEROY, R. (1937) Multiple-stage sewage sludge digestion. Proceedings of American Society Civil Engineers, V. 63, n. 9, p. 1673-1700.

REGY, S.; MANGIN, D.; KLEIN, J.P.; LIETO, J. (2002) Phosphate recovery by struvite precipitation in a stirred reactor. Bruxelas: Centre Européen d’Etudes Des Polyphosphates.

REN, W.; ZHOU, Z.; WAN, L.; HU, D.; JIANG, L.-M.; WANG, L. (2O15) Optimization of phosphorus removal from reject water of sludge thickening and dewatering process through struvite precipitation. Desalination and Water Treatment, v. 57, n. 33, p. 15515-15523. https:// doi.org/10.1080/19443994.2015.1072059

RIVADENEYRA, A.; GONZALEZ-MARTINEZ, A.; GONZALEZ-LOPEZ, J.; MARTIN-RAMOS, D.; MARTINEZ-TOLEDO, M.V:; RIVADENEYRA, M.A. (2014) Precipitation of phosphate minerals by microorganisms isolated from a fixed-biofilm reactor used for the treatment of domestic wastewater. International Journal of Environmental Research and Public Health, v. 11, n. 4, p. 3689-3704. https://dx.doi. org/10.3390\%2Fijerph110403689

RONTELTAP, M.; MAURER, M.; GUJER, W. (2007) Struvite precipitation thermodynamics in source-separated urine. Water Research, v. 41, n. 5, p. 977-984. http://dx.doi.org/10.1016/j.watres.2006.11.046

SCHWARZENBACH, G. (1957) Complexometric titrations. Nova York: Interscience.

SHARMA, B.; SARKAR, A.; SINGH, P.; SINGH, R.P. (2017) Agricultural utilization of biosolids: A review on potential effects on soil and plant grown. Waste Management, v. 64, p. 117-132. https://doi.org/10.1016/j. wasman.2017.03.002

SHIMAMURA, K.; TANAKA, T.; MIURA, Y.; ISHIKAWA, H. (2003) Development of a high-efficiency phosphorus recovery method using a fluidized-bed crystallized phosphorus removal system. Water Science and Technology, v. 48, n. 1, p. 163-170. http://dx.doi.org/10.2166/ wst.2003.0043

SHU, L.; SCHNEIDER, P.; JEGATHEESAN, V.; JOHNSON, J. (2006) An economic evaluation of phosphorus recovery as struvite from digester supernatant. Bioresource Technology, v. 97, n. 17, p. 2211-2216. https://doi.org/10.1016/j.biortech.2005.11.005

SIMÕES, F.; VALE, P.; STEPHENSON, T.; SOARES, A. (2018) The role of pH on the biological struvite production in digested sludge dewatering liquors. Scientific Reports, v. 8, p. 1-9. https://doi.org/10.1038/s41598018-25431-7

SMIT, A.L.; BINDRABAN, P.S.; SCHRÖDER, J.J.; CONIJN, J.G.; VAN DER MEER, H.G. (2009) Phosphorus in agriculture: global resources, trends and developments. Report 282. Wageningen, The Netherlands: Plant Research International. Disponível em: <http://edepot.wur.nl/12571>. Acesso em: 3 dez. 2010.

SMITH, J.A.; CARLIELL-MARQUET, C.M. (2008) The digestibility of iron-dosed activated sludge. Bioresource Technology, v. 99, n. 18, p. 8585-8592. https://doi.org/10.1016/j.biortech.2008.04.005

SOARES, A.; VEESAM, M.; SIMÕES, F.; WOOD, E.; PARSONS, S.A.; STEPHENSON, T. (2014) Bio-Struvite: A new route to recover phosphorus from wastewater. Clean: Soil, Air, Water, v. 42, n. 7. p. 994 997. https://doi.org/10.1002/clen.201300287

SONG, Y.; QIAN, F.; GAO, Y.; HUANG, X.; WU, J.; YU, H. (2O15) PHREEQC program-based simulation of magnesium phosphates crystallization for phosphorus recovery. Environmental Earth Sciences, v. 73, n. 9, p. 5075-5084. https://doi.org/10.1007/s12665-015-4340-8

SONG, Y.; YUAN, P.;ZHENG, B.;PENG, J.; YUAN, F.;GAO, Y.(2OO7)Nutrients removal and recovery by crystallization of magnesium ammonium phosphate from synthetic swine wastewater. Chemosphere, v. 69, n. 2. p. 319-324. https://doi.org/10.1016/j.chemosphere.2007.06.001

STUMM, W:; MORGAN, J.J. (1996) Aquatic chemistry: chemical equilibria and rates in natural waters. Nova York: John Wiley and Sons.

TANSEL, B.; LUNN, G.; MONJE, O. (2018) Struvite formation and decomposition characteristics for ammonia and phosphorus recovery: A review of magnesium-ammonia-phosphate interactions. Chemosphere, v. 194, p. 504-514. https://doi.org/10.1016/j. chemosphere.2017.12.004

TAO, W.; FATTAH, K.P.; HUCHZERMEIER, M.P. (2016) Struvite recovery from anaerobically digested dairy manure: A review of application potential and hindrances. Journal of Environmental Management, v. 169, p. 46-57. http://dx.doi.org/10.1016/j.jenvman.2015.12.006

TAYLOR, A.W.; FRAZIER, A.W.; GURNEY, E.L.; SMITH, J.P. (1963) Solubility products of di- and trimagnesium phosphates and the dissociation of magnesium phosphate solutions. Transactions of the Faraday Society, v. 59, p. 1585-1589. http://dx.doi.org/10.1039/TF9635901585

THISTLETON, J.; BERRY, T.-A.; PEARCE, P.; PARSONS, S.A. (2002) Mechanisms of chemical phosphorus removal II. Iron (III) salts Process Safety and Environmental Protection: Transactions of the Institution of Chemical Engineers, Part B, v. 80, n. 5, p. 265-269. https:// doi.org/10.1205/095758202762277623

TÜRKER, M.; ÇELEN, I. (2010) Chemical equilibrium model of struvite precipitation from anaerobic digester effluents. Turkish Journal of Engineering and Environmental Sciences, v. 34, n. 1, p. 39-48. https:// doi.org/10.3906/muh-1008-15

UENO,Y.;FUJII,M.(2001)Threeyearsexperienceofoperatingandselling recovered struvite from full-scale plant. Environmental Technology, v. 22, n. 11, p. 1373-1381. https://doi.org/10.1080/09593332208618196 
UYSAL, A.; YILMAZEL, Y.D.; DEMIRER, G.N. (2010) The determination of fertilizer quality of the formed struvite from effluent of a sewage sludge anaerobic digester. Journal of Hazardous Materials, v. 181, n. 1-3, p. 248-254. https://doi. org/10.1016/j.jhazmat.2010.05.004

VAN KAUWENBERGH, S.J. (2010) World Phosphate Rock Reserves and Resources. Muscle Shoals: International Fertilizer Development Center (IFDC)

WANG, J.; BURKEN, J.G.; ZHANG, X. (2006) Effect of seeding materials and mixing strength on struvite precipitation. Water Environment Research, v. 78, n. 2, p. 125-132.

WANG, J.; BURKEN, J.G.; ZHANG, X.; SURAMPALLI, R. (2005) Engineered struvite precipitation: Impacts of component-ion molar ratios and pH. Journal of Environmental Engineering, v. 131, n. 10, p. 1433-1440. https://doi.org/10.1061/(ASCE)0733-9372(2005)131:10(1433)

WEBB, K.M.; HO, G.E. (1991) Struvite (MgNH4PO4.6H2O) solubility and its application to a piggery effluent problem. In: WORKSHOP ON APPROPRIATE TECHNOLOGY FOR ENVIRONMENTALLY SUSTAINABLE DEVELOPMENT. Anais... ASEAN, Perth, Australia: Remote Area Developments Group, p. 39-45.

YAN, H.; SHIH, K. (2O16) Effects of calcium and ferric ions on struvite precipitation: A new assessment based on quantitative $\mathrm{X}$-ray diffraction analysis. Water Research, v. 95, p. 310-318. https://doi. org/10.1016/j.watres.2016.03.032

YANG, J.; TIAN, Q.; TAO, F.-F.; LI, Y.-W.; WANG, F.-R. (2O11) Phosphorous precipitation/recovery as struvite from wastewaters of low phosphorous concentration. Journal of Crystal Growth, v. 372, p. 129137. https://doi.org/10.1109/icbbe.2011.5781005

YEOMAN, S.; STEPHENSON, T.; LESTER, J.N.; PERRY, R. (1988) The removal of phosphorus during wastewater treatment: a review. Environmental Pollution, v. 49, n. 3, p. 183-233.

YIGIT, N.Ö:; MAZLUM, S. (2007) Phosphate recovery potential from wastewater by chemical precipitation at batch conditions. Environmental Technology, v. 28, n. 1, p. 83-93. https://doi. org/10.1080/09593332808618768

YU, R.; GENG, J.; REN, H.; WANG, Y.; XU, K. (2O13) Struvite pyrolysate recycling combined with dry pyrolysis for ammonium removal from wastewater. Bioresource Technology, v. 132C, p. 154-159. http://dx.doi. org/10.1016/j.biortech.2013.01.015

ZHANG, T.; DING, L.; REN, H.; XIONG, X. (2009) Ammonium nitrogen removal from coking wastewater by chemical precipitation recycle technology. Water Research, v. 43, n. 20, p. 5209-5215. https://doi. org/10.1016/j.watres.2009.08.054

ZHANG, T., LI, Q.; DING, L.; REN, H.; XU, R.; WU, Y.; SHENG, D. (2O11) Modeling assessment for ammonium nitrogen recovery from wastewater by chemical precipitation. Journal of Environmental Sciences, v. 23, n. 6, p. 881-890. https://doi.org/10.1016/S10010742(10)60485-8

ZHOU, S.; WU, Y. (2012) Improving the prediction of ammonium nitrogen removal through struvite precipitation. Environmental Science Pollution Research, v. 19, n. 2, p. 347-360. http://dx.doi. org/10.1007/s11356-011-0520-6

DOI: 10.1590/S1413-41522019113711ERRATUM

\section{Errata}

No artigo “Nucleação na formação de estruvita: estado da arte”, DOI: 10.1590/S1413-41522019113711, publicado no periódico Eng. Sanit. Ambient. [online]. 2019, vol.24, n.4, p.637-654, nas seguintes páginas:

\section{PÁGINA 641, TABELA 2, LINHA 26}

Onde se lia:

\begin{tabular}{l|l|l}
\hline $\mathrm{Mg}_{3} \mathrm{HPO}_{4} \cdot 3 \mathrm{H}_{2} \mathrm{O} \leftrightarrow 3 \mathrm{H}_{2} \mathrm{O}+\mathrm{HPO}_{4}^{2-}+\mathrm{Mg}^{2+}$ & $-5,8\left(\mathrm{~K}_{\mathrm{PS}}\right)$ & Musvoto et al. (2000), Çelen et al. (2007) \\
\hline
\end{tabular}

Leia-se:

\begin{tabular}{l|l|l}
\hline $\mathrm{MgHPO}_{4} \cdot 3 \mathrm{H}_{2} \mathrm{O} \leftrightarrow 3 \mathrm{H}_{2} \mathrm{O}+\mathrm{HPO}_{4}^{2-}+\mathrm{Mg}^{2+}$ & $-5,8\left(\mathrm{~K}_{\mathrm{PS}}\right)$ & Musvoto et al. (2000), Çelen et al. (2007) \\
\hline
\end{tabular}

\section{PÁGINA 642, TABELA 2, LINHA 1}

\section{Onde se lia:}

\begin{tabular}{|c|c|c|}
\hline $\mathrm{Mg}_{3} \mathrm{HPO}_{4} \cdot 8 \mathrm{H}_{2} \mathrm{O} \leftrightarrow 8 \mathrm{H}_{2} \mathrm{O}+2 \mathrm{PO}_{4}^{3-}+3 \mathrm{Mg}^{2+}$ & $-25,2\left(K_{P S}\right)$ & Musvoto et al. (2000), Mehta e Batstone (2013), Song et al. (2015) \\
\hline \multicolumn{3}{|l|}{ Leia-se: } \\
\hline $\mathrm{Mg}_{3}\left(\mathrm{PO}_{4}\right)_{2} \cdot 8 \mathrm{H}_{2} \mathrm{O} \leftrightarrow 8 \mathrm{H}_{2} \mathrm{O}+2 \mathrm{PO}_{4}^{3-}+3 \mathrm{Mg}^{2+}$ & $-25,2\left(K_{p S}\right)$ & Musvoto et al. (2000), Mehta e Batstone (2013), Song et al. (2015) \\
\hline
\end{tabular}

